Supporting Information for

\title{
Synthesis of Highly Substituted Enantiopure Piperazines and Ketopiperazines from Vicinal $N$-Sulfinyl Diamines.
}

Alma Viso, ${ }^{* \dagger}$ Roberto Fernández de la Pradilla, ${ }^{* \dagger}$ Aida Flores, ${ }^{\dagger}$ Ana García ${ }^{\dagger}$ Mariola Tortosa ${ }^{\dagger}$ and María L. López-Rodríguez."

†Instituto de Química Orgánica General, CSIC, Juan de la Cierva 3, E-28006 Madrid, Spain.

Departamento de Química Orgánica I, Facultad de Ciencias Químicas, Universidad Complutense, E28040 Madrid, Spain. 


\section{TABLE OF CONTENTS}

$\begin{array}{ll}\text { Materials and Methods } & \text { S5 }\end{array}$

1. Synthesis of Starting Materials $\quad$ S5

1.1. (-)-(2S,3R,S $S_{\mathrm{S}}$-2-(Benzylamino)-3-(1-naphtyl)-3-(p-tolylsulfinylamino)propan-1-ol, 1f. $\quad$ S5

1.2. General Procedure for the Protection of N-Sulfinyl Diaminoalcohols 1 as Silyl Ethers, $6 . \quad$ S6

1.2.1. (+)-(1R,2S,S $S_{S}-N$-[2-Benzylamino-3-(t-butyldimethylsilyloxy)-1-ethylprop-1-yl] $p$-tolylsulfinamide, 6a. $\quad$ S6

1.2.2. (+)-(1R,2S, $\left.S_{\mathrm{S}}\right)-N$-[2-benzylamino-3-(t-butyldimethylsilyloxy)-1-(2-phenylethyl)prop-1-yl]

$\begin{array}{ll}\text { p-tolylsulfinamide, } \boldsymbol{6 b} . & \mathrm{S} 7\end{array}$

1.2.3. (+)-(1R,2S,S $\left.S_{\mathrm{S}}\right)-N$-[2-benzylamino-3-(t-butyldimethylsilyloxy)-1-(i-propyl)prop-1-yl] $p$-tolylsulfinamide, $\mathbf{6 c .} \quad \mathrm{S} 7$

1.2.4 (-)-(1R,2S,S $\left.S_{S}\right)-N$-[2-benzylamino-3-(t-butyldimethylsilyloxy)-1-(1-naphtyl)prop-1-yl] $p$-tolylsulfinamide, $\mathbf{6 d .} \quad$ S8

1.2.5. $\quad(+)-\left(1 R, 2 S, \quad S_{\mathrm{S}}\right)-N$-[2-benzylamino-3-(t-butyldimethylsilyloxy)-2-methyl-1-( $i$ propyl)prop-1-yl] $p$-tolylsulfinamide, 6e. S8

2. General Procedure for the Reaction with Diethyl Oxalate S9

2.1. (+)-(5R,6S)-1-Benzyl-5-ethyl-6-hydroxymethylpiperazin-2,3-dione, 2a. S9

2.2. (+)-(5R,6S)-1-Benzyl-6-hydroxymethyl-5-(2-phenylethyl)piperazin-2,3-dione, $2 \mathbf{b} . \quad$ S10

2.3. (+)-(5R,6S)-1-Benzyl-6-hydroxymethyl-5-(i-propyl)piperazin-2,3-dione, 2c. $\quad S 11$

2.4. (+)-(5R,6S)-1-Benzyl-6-hydroxymethyl-5-phenylpiperazin-2,3-dione, 2d. S12

2.5. (+)-(5R,6S)-1-Benzyl-5-(p-fluorophenyl)-6-hydroxymethylpiperazin-2,3-dione, 2e. $\quad$ S12

2.6. (-)-(5R,6S)-1-Benzyl-6-hydroxymethyl-5-(1-naphtyl)piperazine-2,3-dione, 2f. $\quad$ S13

3. General Procedure of Reduction with $\mathrm{BH}_{3} \cdot \mathbf{S M e}_{2}$.

3.1. (-)-(2S,3R)-1-Benzyl-3-ethyl-2-hydroxymethylpiperazine, 4a. S14

3.2. (+)-(2S,3R)-1-Benzyl-2-hydroxymethyl-3-(2-phenylethyl)piperazine, 4b. S14

3.3. (-)-(2S,3R)-1-Benzyl-2-hydroxymethyl-3-i-propylpiperazine, 4c. S15

3.4. (-)-(2S,3R)-1-Benzyl-2-hydroxymethyl-3-phenylpiperazine, 4d. S15

3.5. (-)-(2S,3R)-1-Benzyl-3-p-fluorophenyl-2-hydroxymethylpiperazine, 4e. S16

4.Synthesis of (-)-(2R,3S)-4-benzyl-1-benzyloxycarbonyl-2-ethyl-3-hydroxymethylpiperazine, 5.

5. General Procedure for the Synthesis of Chloroacetamides. S17

5.1. (+)- $N$-Benzyl- $N-\left[\left(1 S, 2 R, S_{\mathrm{S}}\right)-1\right.$-(t-butyldimethylsilyloxymethyl)-2-(p-tolylsulfinylamino)but-1-yl] 2-chloroacetamide,

7a.

5.2. (+)- $N$-Benzyl- $N$ - $\left[\left(1 S, 2 R, S_{\mathrm{S}}\right)-1\right.$-(t-butyldimethylsilyloxymethyl)-4-phenyl-2-(p-

tolylsulfinylamino)but-1-yl] 2-chloroacetamide, $\mathbf{7 b}$. 
5.3. $\quad(+)-N$-Benzyl- $N-\left[\left(1 S, 2 R, S_{\mathrm{S}}\right)-1-(t\right.$-butyldimethylsilyloxymethyl)-3-methyl-2- $(p-$ tolylsulfinylamino)but-1-yl]-2-chloroacetamide, 7c.

5.4. (+)- $N$-Benzyl- $N-\left[\left(1 S, 2 R, S_{\mathrm{S}}\right)-1-(t\right.$-butyldimethylsilyloxymethyl)-2-(1-naphtyl)-2- $(p$ tolylsulfinylamino)eth-1-yl]-2-chloroacetamide, 7d.

5.5. (+)- $N$-Benzyl- $N$-[(1S,2R, $\left.S_{\mathrm{S}}\right)$-1-(hydroxymethyl)-2-(1-naphtyl)-2-(p-tolylsulfinylamino)eth1-yl]-2-chloroacetamide, 7e.

5.6. $\quad(+)$-Methyl $\quad\left[\left(2 S, 3 R, S_{\mathrm{S}}\right)-2\right.$-chloroacetylamino-3- $(p$-fluorophenyl $)-3-(p$ -

tolylsulfinylamino)]propanoate, 11a.

5.7. ( \pm -Methyl $\left[\left(2 S, 3 R, S_{\mathrm{S}}\right)-2\right.$-chloroacetylamino-4-methyl-3-(p-tolylsulfinylamino)]pentanoate, 11b. $\quad$ S20

5.8. ( \pm )-Methyl $\left[\left(2 S, 3 R, S_{\mathrm{S}}\right)\right.$-2-chloroacetylamino-3-(p-tolylsulfinylamino) $]$ butanoate, 11c. $\quad$ S21

\section{General Procedure for the Synthesis of Ketopiperazines.}

6.1.

(+)-(5R,6S,SS)-1-Benzyl-6-[( $t$-butyldimethylsilyloxy)methyl]-5-ethyl-4- $(p$ -

tolylsulfinyl)piperazin-2-one, $\mathbf{8 a}$.

6.2. (+)-(5R,6S,SS)-1-Benzyl-6-[( $t$-butyldimethylsilyloxy)methyl]-5-(2-phenylethyl)-4- $(p$ tolylsulfinyl)piperazin-2-one, $\mathbf{8 b}$.

6.3. $\quad(+)-(5 R, 6 S, S S)-1-B e n z y l-6-[(t$-butyldimethylsilyloxy)methyl]-5-( $i$-propyl)-4- $(p$ tolylsulfinyl)piperazin-2-one, $\mathbf{8 c}$.

6.4. $\quad(-)-(5 R, 6 S, S S)-1-B e n z y l-6-[(t$-butyldimethylsilyloxy)methyl]-5-(1-naphtyl)-4- $(p$ tolylsulfinyl)piperazin-2-one, $8 \mathbf{d}$.

6.5. (+)-Methyl [(2S,3R,SS)-3-(p-fluorophenyl)-6-oxo-4-(p-tolylsulfinyl)piperazin-2-yl] carboxylate, 12.

6.6. (-)-(5R,6S,SS)-1-Benzyl-6-hydroxymethyl-5-(1-naphtyl)-4-(p-tolylsulfinyl)piperazin-2one, 8 e.

7. General Procedure for the Synthesis of Imino Ketopiperazines 9.

7.1. (+)-(5R,6S)-1-Benzyl-6-( $t$-butyldimethylsilyloxymethyl)-5-ethyl-5,6-dihydro-1H-pyrazin2-one, 9a.

7.2. (+)-(5R,6S)-1-Benzyl-6-(t-butyldimethylsilyloxymethyl)-5-(2-phenylethyl)-5,6-dihydro$1 H$-pyrazin-2-one, $9 \mathbf{b}$.

7.3. (+)-(5R,6S)-1-Benzyl-6-( $t$-butyldimethylsilyloxymethyl)-5-( $i$-propyl)-5,6-dihydro-1Hpyrazin-2-one, $9 \mathbf{c}$.

7.4. (+)-(5R,6S)-1-Benzyl-6-( $t$-butyldimethylsilyloxymethyl)-5-(1-naphtyl)-5,6-dihydro-1Hpyrazin-2-one, 9d. 
8.1. General Procedure for the Addition of Potassium Cyanide.

S28

8.1.1.

$(5 S, 6 R)-4-B e n z y l-5-[(t$-butyldimethylsilyloxy)methyl]-3-oxo-6-(2phenylethyl)piperazin-2-yl]carbonitrile, 13a and 14a.

8.1.2. (5S,6R)-4-Benzyl-5-[( $t$-butyldimethylsilyloxy)methyl]-3-oxo-6-( $i$-propyl)piperazin-2yl]carbonitrile, $\mathbf{1 3 b}$ and $\mathbf{1 4 b .}$

8.2. Addition of Diethylzinc.

$(3 S, 5 R, 6 S)$-1-Benzyl-6-[(t-butyldimethylsilyloxy)methyl]-3-ethyl-5-(2-phenylethyl)piperazin2-one, 13c and (3R,5R,6S)-1-Benzyl-6-[(t-butyldimethylsilyloxy)methyl]-3-ethyl-5-(2phenylethyl)piperazin-2-one, 14c.

8.3. Allylations.

8.3.1. Procedure of Addition of allyltributylstannane and $\mathrm{TiCl}_{4}$.

8.3.2. Procedure of Addition of allyltrimethylsilane and $\mathrm{SnCl}_{4}$.

8.3.3. Procedure of Addition of Allylmagnesium Bromide and $\mathrm{BF}_{3} \cdot \mathrm{OEt}_{2}$.

8.3.4. Procedure of Addition of Allylmagnesium Bromide and $\mathrm{CeCl}_{3}$.

8.3.5. Procedure for Zinc-Mediated Barbier Allylation.

(+)-(3R,5R,6S)-3-Allyl-1-benzyl-6-[( $t$-butyldimethylsilyloxy)methyl]-5-( $i$-propyl)piperazin2-one, 14d.

(-)-(3S,5R,6S)-3-Allyl-1-benzyl-6-[( $t$-butyldimethylsilyloxy)methyl]-5-( $i$-propyl)piperazin2-one, 13d.

(+)-(3R,5S,6R)-1-Benzyl-6-[( $t$-butyldimethylsilyloxy)methyl]-3-(1,1-dimethylallyl)-5-( $i$ propyl)piperazin-2-one, 14e and (3R,5S,6R)-1-benzyl-6-[( $t$-butyldimethylsilyloxy)methyl]3-(3-methylbut-2-enyl)-5-( $i$-propyl)piperazin-2-one $\mathbf{1 4 f}$. 
Materials and Methods. All reactions were carried out under a positive pressure of dry argon, using freshly distilled solvents under anhydrous conditions. Reagents and solvents were handled by using standard syringe techniques. $\mathrm{Et}_{2} \mathrm{O}$ and THF were distilled from sodium and benzophenone, $\mathrm{CH}_{2} \mathrm{Cl}_{2}$ was distilled from $\mathrm{CaH}_{2}$. Crude products were purified by flash chromatography on 230-400 mesh silica gel with distilled solvents. Analytical TLC was carried out on silica gel plates. Through this section, the volume of solvents is reported in $\mathrm{mL} / \mathrm{mmol}$ of starting material. ${ }^{1} \mathrm{H}$ and ${ }^{13} \mathrm{C} \mathrm{NMR}$ spectra were recorded on $200 \mathrm{MHz}, 300 \mathrm{MHz}, 400 \mathrm{MHz}$ and $500 \mathrm{MHz}$ spectrometers using $\mathrm{CDCl}_{3}$ as solvent and with the residual solvent signal as internal reference $\left(\mathrm{CDCl}_{3}, 7.24\right.$ and $\left.77.0 \mathrm{ppm}\right)$ unless otherwise noted. The following abbreviations are used to describe peak patterns when appropriate: $s$ (singlet), $d$ (doublet), $t$ (triplet), q (quartet), quint (quintuplet), sext (sextet), m (multiplet), ap (apparent), br (broad). Melting points are uncorrected. Optical rotations were measured on a polarimeter at $20^{\circ} \mathrm{C}$ using a sodium lamp and in $\mathrm{CHCl}_{3}$ solution. Low resolution mass spectra were recorded by direct injection using the electronic impact technique with an ionization energy of $70 \mathrm{eV}(\mathrm{EI})$ or using the atmospheric pressure chemical ionization (APCI) or electrospray (ES) chemical ionization techniques in its positive or negative modes. Elemental analyses were carried out at Instituto de Química Orgánica, CSIC (Madrid).

\section{Synthesis of Starting Materials.}

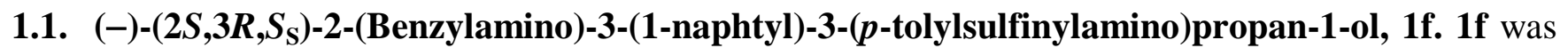
prepared from a suspension of $\mathrm{LiAlH}_{4}(81 \mathrm{mg}, 2.124 \mathrm{mmol})$ in $\mathrm{Et}_{2} \mathrm{O}$ and methyl $\left[\left(2 S, 4 S, 5 R, S_{\mathrm{S}}\right)-5-(1-\right.$ naphtyl)-2-phenyl-1-(p-tolylsulfinyl)-1,3-imidazolidin-4-yl]carboxylate $\quad(250 \quad \mathrm{mg}, \quad 0.531 \quad \mathrm{mmol})$,

according to a reported procedure ${ }^{1}(4 \mathrm{~h}) . \mathbf{1 f}$ was isolated as a white foam (190 $\left.\mathrm{mg}, 0.428 \mathrm{mmol}, 81 \%\right)$ after purification by column chromatography $\left(0-2 \% \mathrm{MeOH}-\mathrm{CH}_{2} \mathrm{Cl}_{2}\right)$. Data for $\mathbf{1 f}: \boldsymbol{R}_{\mathbf{f}}=0.24(3 \%$ $\left.\mathrm{MeOH}-\mathrm{CH}_{2} \mathrm{Cl}_{2}\right) . \mathbf{m p}: 43-45^{\circ} \mathrm{C} .[\alpha]^{\mathbf{2 0}}{ }_{\mathbf{D}}=-68.3(c=1.24) .{ }^{\mathbf{1}} \mathbf{H}$ NMR $(300 \mathrm{MHz}) \delta 1.50($ br s), $2.01(\mathrm{~s}$ $3 \mathrm{H}), 3.10(\mathrm{ddd}, 1 \mathrm{H}, J=8.2,5.4,2.9 \mathrm{~Hz}), 3.18(\mathrm{~d}, 1 \mathrm{H}, J=12.8 \mathrm{~Hz}), 3.30(\mathrm{~d}, 1 \mathrm{H}, J=12.8 \mathrm{~Hz}), 3.60$ $(\mathrm{dd}, 1 \mathrm{H}, J=11.7,8.8 \mathrm{~Hz}), 3.68(\mathrm{~m}, 1 \mathrm{H}), 5.32(\mathrm{br} \mathrm{d}, 1 \mathrm{H}, J=6.1 \mathrm{~Hz}), 5.96(\mathrm{~d}, 1 \mathrm{H}, J=7.1 \mathrm{~Hz}), 6.78$ (d, $2 \mathrm{H}, J=8.3 \mathrm{~Hz}), 6.88(\mathrm{br} \mathrm{d}, 2 \mathrm{H}, J=2.7 \mathrm{~Hz}), 7.13(\mathrm{~m}, 3 \mathrm{H}), 7.28-7.40$ (m, $5 \mathrm{H})$, 7.64-7.75 (m, $4 \mathrm{H})$. 
${ }^{13}$ C NMR (50 MHz) $\delta$ 20.9, 48.4, 52.1, 61.0, 61.3, 122.7, 124.8, 125.3, 125.9 (2 C), 127.0 (2 C), 127.5, 127.7 (2 C), 128.3 (2 C), 128.5 (2 C), 128.9, 129.7 (2 C), 133.5, 137.2, 138.7, 139.7, 140.9. IR $(\mathrm{KBr}): v=3307,3057,2927,2869,1597,1495,1454,1262,1087,1058,799,738,698 \mathrm{~cm}^{-1}$. MS (ES): $445[\mathrm{M}+1]^{+}(100 \%)$. Anal. calcd for $\mathrm{C}_{20} \mathrm{H}_{21} \mathrm{~N}_{2} \mathrm{O}_{2} \mathrm{~S}$ (353.1): C 67.96, H 5.99, N 7.93, S 9.07; found: C 67.55, H 6.02, N 7.77, S 9.14.

\subsection{General Procedure for the Protection of $N$-Sulfinyl Diaminoalcohols 1 as Silyl Ethers 6. A} solution of the $N$-sulfinyl diaminoalcohol, 2 equiv of TBDMSCl, 2 equiv of imidazole and 0.05 equiv of DMAP in $\mathrm{CH}_{2} \mathrm{Cl}_{2}(5 \mathrm{~mL} / \mathrm{mmol})$ was stirred at room temperature and monitored by TLC until disappearance of the starting material $(1-4 \mathrm{~h})$. Then, water $(10 \mathrm{~mL} / \mathrm{mmol})$ was added and the layers were separated. The aqueous layer was washed twice with $\mathrm{CH}_{2} \mathrm{Cl}_{2}(5 \mathrm{~mL} / \mathrm{mmol})$. The combined organic extracts were washed with a saturated solution of $\mathrm{NaCl}$, dried over $\mathrm{Na}_{2} \mathrm{SO}_{4}$ and filtered to give, after evaporation of the solvent, a crude product that was purified by chromatography on silica gel using the appropriate mixture of solvents as eluent.

\subsection{1. $(+)-\left(1 R, 2 S, S_{\mathrm{S}}\right)-N$-[2-Benzylamino-3-(t-butyldimethylsilyloxy)-1-ethylprop-1-yl] $\quad p$ -}

tolylsulfinamide, 6a. 6a (163 mg, $0.352 \mathrm{mmol}, 88 \%)$ was obtained as a colorless oil from 1a (140 mg, $0.40 \mathrm{mmol}$ ) following the general procedure (1 h $30 \mathrm{~min}$ ) and after purification by chromatography (50100\% $\mathrm{Et}_{2} \mathrm{O}$-hexane). Data for 6a: $\boldsymbol{R}_{\mathbf{f}}=0.32\left(\mathrm{Et}_{2} \mathrm{O}\right) \cdot[\alpha]^{\mathbf{2 0}}{ }_{\mathbf{D}}=+62.9(c=1.14) .{ }^{1} \mathbf{H} \mathbf{N M R}(300 \mathrm{MHz}) \delta$ $0.04(\mathrm{~s}, 3 \mathrm{H}), 0.05(\mathrm{~s}, 3 \mathrm{H}), 0.77$ (t, $3 \mathrm{H}, J=7.4 \mathrm{~Hz}), 0.87(\mathrm{~s}, 9 \mathrm{H}), 1.41-1.64(\mathrm{~m}, 3 \mathrm{H}), 2.38(\mathrm{~s}, 3 \mathrm{H})$, $2.80(\mathrm{td}, 1 \mathrm{H}, J=6.2,3.5 \mathrm{~Hz}), 3.27(\mathrm{qd}, 1 \mathrm{H}, J=7.0,3.5 \mathrm{~Hz}), 3.64(\mathrm{~d}, 2 \mathrm{H}, J=6.2 \mathrm{~Hz}), 3.75(\mathrm{~d}, 1 \mathrm{H}, J$ $=12.9 \mathrm{~Hz}), 3.86(\mathrm{~d}, 1 \mathrm{H}, J=12.9 \mathrm{~Hz}), 4.65(\mathrm{~d}, 1 \mathrm{H}, J=7.6 \mathrm{~Hz}), 7.21-7.29(\mathrm{~m}, 7 \mathrm{H}), 7.56(\mathrm{~d}, 2 \mathrm{H}, J=$ $8.3 \mathrm{~Hz}) .{ }^{13} \mathrm{C}$ NMR $(50 \mathrm{MHz}) \delta-5.4(2 \mathrm{C}), 10.6,18.1,21.3,25.9,52.8,56.4,60.3,62.8,125.6(2 \mathrm{C})$, 127.0, 128.2 (2 C), 128.3 (2 C), 129.3 (2 C), 140.7, 140.9, 142.8. IR (film): $v=3307,3027,2928$, 2856, 1493, 1462, 1255, 1089, 1067, 837, 811, 776, $698 \mathrm{~cm}^{-1}$. MS (ES): $463[\mathrm{M}+3]^{+}, 462[\mathrm{M}+2]^{+}$, $461[\mathrm{M}+1]^{+}(100 \%)$. 
1.2.2. (+)-(1R,2S, $\left.S_{\mathrm{S}}\right)-N$-[2-benzylamino-3-(t-butyldimethylsilyloxy)-1-(2-phenylethyl)prop-1-yl] $p$ -

tolylsulfinamide 6b. $6 b(283 \mathrm{mg}, 0.528 \mathrm{mmol}, 68 \%)$ was obtained as a colorless oil from $\mathbf{1 b}(330 \mathrm{mg}$, $0.781 \mathrm{mmol})$ following the general procedure $(4 \mathrm{~h})$ and after purification by chromatography $(20-40 \%$ $\mathrm{Et}_{2} \mathrm{O}-$ hexane $)$. Data for $\mathbf{6 b}: \boldsymbol{R}_{\mathbf{f}}=0.24\left(70 \% \mathrm{Et}_{2} \mathrm{O}\right.$-hexane $) . \quad[\alpha]^{\mathbf{2 0}}{ }_{\mathbf{D}}=+53.4(\mathrm{c}=2.80) .{ }^{\mathbf{1}} \mathbf{H}$ NMR $(300$ MHz) $\delta 0.04(\mathrm{~s}, 3 \mathrm{H}), 0.05$ (s, $3 \mathrm{H}), 0.87$ (s, $9 \mathrm{H}), 1.41$ (br s, $1 \mathrm{H}), 1.67-1.91$ (m, $2 \mathrm{H}), 2.34-2.44$ (m, 1 H), $2.40(\mathrm{~s}, 3 \mathrm{H}), 2.52-2.61(\mathrm{~m}, 1 \mathrm{H}), 2.90(\mathrm{td}, 1 \mathrm{H}, J=6.2,3.4 \mathrm{~Hz}), 3.39(\mathrm{qd}, 1 \mathrm{H}, J=6.7,3.4 \mathrm{~Hz})$, $3.64(\mathrm{~d}, 2 \mathrm{H}, J=6.1 \mathrm{~Hz}), 3.72(\mathrm{~d}, 1 \mathrm{H}, J=12.9 \mathrm{~Hz}), 3.83(\mathrm{~d}, 1 \mathrm{H}, J=12.9 \mathrm{~Hz}), 4.84(\mathrm{~d}, 1 \mathrm{H}, J=7.3$ Hz), $7.01(\mathrm{~d}, 2 \mathrm{H}, J=7.1 \mathrm{~Hz}), 7.14(\mathrm{~d}, 1 \mathrm{H}, J=7.1 \mathrm{~Hz}), 7.20(\mathrm{~d}, 2 \mathrm{H}, J=7.6 \mathrm{~Hz}), 7.23-7.30(\mathrm{~m}, 7 \mathrm{H})$, $7.59(\mathrm{~d}, 2 \mathrm{H}, J=8.1 \mathrm{~Hz}) .{ }^{13} \mathrm{C}$ NMR $(75 \mathrm{MHz}) \delta-4.8(2 \mathrm{C}), 18.8,22.0,26.6$ (3 C), 32.9, 35.1, 53.4, 54.8, 61.5, 63.4, 126.3 (2 C), 126.4 (2 C), 127.7 (2 C), 128.9 (2 C), 129.0 (2 C), 129.1 (2 C), 130.1 (2 C), 141.3, 141.7, 142.5, 143.3. IR (film): $v=3304,3219,3084,3061,3026,2952,2928,2884,2856$, $1943,1804,1602,1494,1470,1454,1400,1360,1300,1255,1217,1177,1089,1067,1017,1006$, 939, 837, 812, 777, 751, 699, 667, $621 \mathrm{~cm}^{-1}$. MS (ES): $537[\mathrm{M}+1]^{+}(100 \%)$.

\subsection{3. (+)-(1R,2S, $\left.S_{\mathrm{S}}\right)-N$-[2-benzylamino-3-(t-butyldimethylsilyloxy)-1-(i-propyl)prop-1-yl] $\quad p$ -}

tolylsulfinamide 6c. 6c $(85 \mathrm{mg}, 0.179 \mathrm{mmol}, 64 \%)$ was obtained as a colorless oil from a solution of 1c $(101 \mathrm{mg}, 0.281 \mathrm{mmol})$ following the general procedure $(3 \mathrm{~h})$ and after purification by chromatography (20-40\% $\mathrm{Et}_{2} \mathrm{O}$-hexane). Data for $\mathbf{9 c}: \boldsymbol{R}_{\mathbf{f}}=0.14\left(70 \% \mathrm{Et}_{2} \mathrm{O}\right.$-hexane $) .[\alpha]^{\mathbf{2 0}}{ }_{\mathbf{D}}=+44.7(c$ = 2.91). ${ }^{1} \mathbf{H}$ NMR $(300 \mathrm{MHz}) \delta 0.04(\mathrm{~s}, 3 \mathrm{H}), 0.05(\mathrm{~s}, 3 \mathrm{H}), 0.76(\mathrm{~d}, 3 \mathrm{H}, J=6.8 \mathrm{~Hz}), 0.82(\mathrm{~d}, 3 \mathrm{H}, J=$ $6.8 \mathrm{~Hz}), 0.87(\mathrm{~s}, 9 \mathrm{H}), 1.75(\mathrm{~m}, 1 \mathrm{H}), 1.44(\mathrm{br} \mathrm{s}, 1 \mathrm{H}), 2.37(\mathrm{~s}, 3 \mathrm{H}), 2.71$ (ddd, $1 \mathrm{H}, J=7.2,5.7,2.9$ Hz), 3.07 (ddd, $1 \mathrm{H}, J=8.5,5.6,2.9 \mathrm{~Hz}), 3.64(\mathrm{~m}, 2 \mathrm{H}), 3.73(\mathrm{~d}, 1 \mathrm{H}, J=12.9 \mathrm{~Hz}), 3.92(\mathrm{~d}, 1 \mathrm{H}, J=$ $12.9 \mathrm{~Hz}), 4.65(\mathrm{~d}, 1 \mathrm{H}, J=8.5 \mathrm{~Hz}), 7.23(\mathrm{~m}, 3 \mathrm{H}), 7.29(\mathrm{~m}, 4 \mathrm{H}), 7.57(\mathrm{~d}, 2 \mathrm{H}, J=8.1 \mathrm{~Hz}) .{ }^{13} \mathbf{C} \mathbf{N M R}$ (75 MHz) $\delta-5.4(2 \mathrm{C}), 18.1,18.7,19.7,21.3,25.9$ (3 C), 31.3, 52.7, 59.0, 59.5, 63.2, 125.7 (2 C), 127.0, 128.2 (2 C), 128.3 (2 C), 129.3 (2 C), 140.7, 141.0, 142.6. IR (film): $v=3311,3028,2955$, 2928, 2856, 1598, 1493, 1470, 1388, 1361, 1255, 1089, 939, 837, 811, 776, 738, $699 \mathrm{~cm}^{-1}$. MS (ES): $475[\mathrm{M}+1]^{+}(100 \%), 335[\mathrm{M}-(p \text { TolSO })]^{+1}$. 


\subsection{4 (-)-(1R,2S, $\left.S_{\mathrm{S}}\right)-N$-[2-benzylamino-3-(t-butyldimethylsilyloxy)-1-(1-naphtyl)prop-1-yl] $\quad p$ -}

tolylsulfinamide 6d. 6d (106 mg, $0.190 \mathrm{mmol}$, 78\%) was obtained as a colorless oil from $\mathbf{1 f}$ (109 mg, $0.245 \mathrm{mmol})$ following the general procedure $(3 \mathrm{~h})$ and after purification by chromatography $(30-40 \%$ $\mathrm{Et}_{2} \mathrm{O}$-hexane). Data for $\mathbf{6 d}: \boldsymbol{R}_{\mathbf{f}}=0.32\left(70 \%\right.$ EtOAc-hexane). $[\alpha]^{\mathbf{2 0}}{ }_{\mathbf{D}}=-17.6(c=0.62) .{ }^{\mathbf{1}} \mathbf{H} \mathbf{~ N M R}(300$ MHz) $\delta-0.06(\mathrm{~s}, 3 \mathrm{H}),-0.04(\mathrm{~s}, 3 \mathrm{H}), 0.89$ (s, $9 \mathrm{H}), 1.73($ br s, $1 \mathrm{H}), 2.00$ (s, $3 \mathrm{H}), 3.04(\mathrm{~m}, 1 \mathrm{H}), 3.27$ $(\mathrm{dm}, 1 \mathrm{H}, J=7.8 \mathrm{~Hz}), 3.55(\mathrm{~d}, 1 \mathrm{H}, J=12.7 \mathrm{~Hz}), 3.66(\mathrm{~m}, 1 \mathrm{H}), 3.75(\mathrm{~d}, 1 \mathrm{H}, J=12.0 \mathrm{~Hz}), 5.23(\mathrm{~m}, 1$ H), $6.58(\mathrm{~s}, 1 \mathrm{H}), 6.58(\mathrm{~d}, 2 \mathrm{H}, J=7.1 \mathrm{~Hz}), 7.12-7.38(\mathrm{~m}, 11 \mathrm{H}), 7.53(\mathrm{~d}, 1 \mathrm{H}, J=8.1 \mathrm{~Hz}), 7.66(\mathrm{~d}, 1 \mathrm{H}$, $J=7.6 \mathrm{~Hz}), 7.95(\mathrm{~d}, 1 \mathrm{H}, J=8.1 \mathrm{~Hz}) .{ }^{13} \mathrm{C} \mathbf{N M R}(50 \mathrm{MHz}) \delta-5.6,-5.5,18.2,20.9,25.9(3 \mathrm{C}), 51.5(2$ C), 60.1 (2 C), 124.8, 125.1, 125.3 (3 C), 127.1 (2 C), 127.2, 128.1 (2 C), 128.2 (3 C), 128.3 (2 C), 128.4 (2 C), 130.9, 133.5, 139.8, 140.1 (2 C). MS (ES): $1339[2 \mathrm{M}+\mathrm{Na}]^{+}, 581[\mathrm{M}+\mathrm{Na}]^{+}, 559[\mathrm{M}+1]^{+}$ $(100 \%)$.

\subsection{5. (+)-(1R,2S, $\left.S_{\mathrm{S}}\right)-N$-[2-benzylamino-3-(t-butyldimethylsilyloxy)-2-methyl-1-(i-propyl)prop-1-}

yl] $p$-tolylsulfinamide, 6e. 6e $(54 \mathrm{mg}, 0.11 \mathrm{mmol}, 75 \%)$ was obtained as a colorless oil from $1 \mathrm{~g}(55$ $\mathrm{mg}, 0.15 \mathrm{mmol})$ following the general procedure $(3 \mathrm{~h})$ and after purification by chromatography (30$100 \% \mathrm{Et}_{2} \mathrm{O}$-hexane). Data for $\mathbf{6 e}: \boldsymbol{R}_{\mathbf{f}}=0.28\left(80 \% \mathrm{Et}_{2} \mathrm{O}\right.$-hexane $) .[\alpha]^{\mathbf{2 0}}{ }_{\mathbf{D}}=+14.8(c=1.28) .{ }^{\mathbf{1}} \mathbf{H} \mathbf{N M R}$ $(300 \mathrm{MHz}) \delta-0.02(\mathrm{~s}, 3 \mathrm{H}), 0.08(\mathrm{~s}, 3 \mathrm{H}), 0.78(\mathrm{~s}, 9 \mathrm{H}), 0.92(\mathrm{~d}, 3 \mathrm{H}, J=6.9 \mathrm{~Hz}), 1.09(\mathrm{~d}, 3 \mathrm{H}, J=6.9$ Hz), 1.19 (s, 3 H), 2.15 (m, 1 H), 2.37 (s, 3 H), 3.39 (d, $1 \mathrm{H}, J=9.6 \mathrm{~Hz}), 3.44$ (d, $1 \mathrm{H}, J=9.9 \mathrm{~Hz}), 3.60$ $(\mathrm{d}, 1 \mathrm{H}, J=9.9 \mathrm{~Hz}), 3.72(\mathrm{AB}$ system, $2 \mathrm{H}), 4.44(\mathrm{~d}, 1 \mathrm{H}, J=9.6 \mathrm{~Hz}), 7.23$ (d, $2 \mathrm{H}, J=8.1 \mathrm{~Hz}), 7.26-$ $7.30(\mathrm{~m}, 5 \mathrm{H}), 7.65(\mathrm{~d}, 2 \mathrm{H}, J=8.1 \mathrm{~Hz}) .{ }^{13} \mathbf{C} \mathbf{N M R}(50 \mathrm{MHz}) \delta-5.7,-5.6,17.8,17.9,20.4,21.3$, 23.2, 25.7 (3 C), 26.4, 46.2, 59.9, 66.0, 67.0, 125.4 (2 C), 126.9, 128.1 (2 C), 128.4 (2 C), 129.3 (2 C), 140.9 (2 C), 144.2. IR (film): $v=3341,3021,2960,2927,2855,1470,1259,1091,836,810,698$ $\mathrm{cm}^{-1}$. MS (ES): $491[\mathrm{M}+3]^{+}, 490[\mathrm{M}+2]^{+}, 489[\mathrm{M}+1]^{+}(100 \%)$. Anal. calcd for $\mathrm{C}_{27} \mathrm{H}_{44} \mathrm{~N}_{2} \mathrm{O}_{2} \mathrm{SSi}$ (488.8): C, 66.34; H, 9.07; N, 5.73; S, 6.56; Si, 5.75; found: C, 66.55; H, 9.19; N, 5.81; S, 6.77. 
2. General Procedure for the Reaction with Diethyl Oxalate. Method A: To a solution of $N$ sulfinyldiaminoalcohol 1 in anhydrous $\mathrm{CH}_{2} \mathrm{Cl}_{2}(6 \mathrm{~mL} / \mathrm{mmol})$ at room temperature, 6 equiv of diethyl oxalate was added and the mixture was stirred until formation of morpholinedione $\mathbf{3}$ was observed by TLC. Then, 2 equiv of $\mathrm{NaOMe}$ (from a $0.25 \mathrm{M}$ solution in $\mathrm{MeOH}$ ) was added and the mixture was stirred until disappearance of starting materials (TLC). At that point, water $(10 \mathrm{~mL} / \mathrm{mmol})$ was added, the solvents $\left(\mathrm{MeOH} / \mathrm{CH}_{2} \mathrm{Cl}_{2}\right)$ were evaporated in vacuo. The residue was diluted with water $(10$ $\mathrm{mL} / \mathrm{mmol}$ ) and extracted with $\mathrm{CH}_{2} \mathrm{Cl}_{2}$ (3 times, $5 \mathrm{~mL} / \mathrm{mmol}$ ). The combined organic extracts were dried over $\mathrm{Na}_{2} \mathrm{SO}_{4}$ and filtered to give, after evaporation of the solvent, a crude product that was purified by column chromatography on silica gel using the appropriate mixture of solvents. Usually piperazin-2,3diones 2, precipitate in $\mathrm{CH}_{2} \mathrm{Cl}_{2}$ and $\mathrm{Et}_{2} \mathrm{O}$ therefore they could be purified by washing repeatedly the crude solid product with $90 \% \mathrm{Et}_{2} \mathrm{O}$-hexane. Method B: To a solution of diaminoalcohol $\mathbf{1}$ at room temperature in anhydrous $\mathrm{CH}_{2} \mathrm{Cl}_{2}(6 \mathrm{~mL} / \mathrm{mmol}), 6$ equiv of diethyl oxalate was added. The mixture was stirred until formation of morpholinedione $\mathbf{3}$ and piperazin-2,3-dione $\mathbf{2}$ was observed by TLC. Then, the solvent was removed under reduced pressure and the piperazin-2,3-dione was isolated by washing thoroughly the crude product with $\mathrm{CH}_{2} \mathrm{Cl}_{2}$ or by column chromatography using the appropriate mixture of solvents. The mother liquors or the chromatographic fraction containing the morpholinedione $\mathbf{3}$ was treated with 2 equiv of $\mathrm{NaOMe}$ (from a $0.25 \mathrm{M}$ solution in $\mathrm{MeOH}$ ) until no starting material was detected by TLC. Isolation and purification of piperazin-2,3-diones 2 was carried out as described in method A. 3a was the only morpholinedione fully characterized.

2.1. (+)-(5R,6S)-1-Benzyl-5-ethyl-6-hydroxymethylpiperazin-2,3-dione, 2a. From 1a (416 mg, 1.20 mmol), diethyl oxalate $(0.98 \mathrm{~mL}, 7.20 \mathrm{mmol})$ and 0.5 equiv of a solution of $\mathrm{NaOMe}$ in $\mathrm{MeOH}(0.3 \mathrm{M}$, $2 \mathrm{~mL}, 0.60 \mathrm{mmol}$ ), following general procedure A, diketopiperazine 2a was obtained. Purification by washing thoroughly the crude product with $90 \% \mathrm{Et}_{2} \mathrm{O}$-hexane and chromatography $(5 \% \mathrm{MeOH}-$ $\left.\mathrm{CH}_{2} \mathrm{Cl}_{2}\right)$ of the mother liquor afforded $250 \mathrm{mg}(0.95 \mathrm{mmol}, 79 \%)$ of $\mathbf{2 a}$ as a white solid. In a previous experiment from 1a $(132 \mathrm{mg}, 0.38 \mathrm{mmol})$ and diethyl oxalate $(0.31 \mathrm{~mL}, 2.28 \mathrm{mmol})$, following general 
procedure $\mathbf{B}(40 \mathrm{~h})$, without adding $\mathrm{NaOMe}$ in $\mathrm{MeOH}$, a mixture of diketopiperazine 2a and (+)$\left(1 R, S_{\mathrm{S}}\right)$-p-toluenesulfinic acid [1-(4-benzyl-5,6-dioxomorpholin-3-(S)-yl)-propyl]amide, $3 \mathbf{3 a}$ was obtained. A white solid was separated from the reaction mixture by filtration and was washed with $\mathrm{CH}_{2} \mathrm{Cl}_{2}$ to afford $60 \mathrm{mg}(0.23 \mathrm{mmol}, 60 \%)$ of $\mathbf{2 a}$ as a white solid. Two consecutive chromatographies of the mother liquors $\left(\mathrm{CH}_{2} \mathrm{Cl}_{2}\right.$ to $25: 1 \mathrm{CH}_{2} \mathrm{Cl}_{2}-\mathrm{MeOH}$ and $20: 1 \mathrm{CH}_{2} \mathrm{Cl}_{2}$-EtOH $)$ afforded $23 \mathrm{mg}(0.057$ mmol, $15 \%$ ) of pure 3a as a white foam which was crystallized from $75 \% \mathrm{Et}_{2} \mathrm{O}$-hexane. Data for $\mathbf{2 a}$ : mp: $90-92{ }^{\circ} \mathrm{C} . \quad \boldsymbol{R}_{\mathbf{f}}=0.25\left(12: 1 \quad \mathrm{CH}_{2} \mathrm{Cl}_{2}-\mathrm{MeOH}\right) . \quad[\alpha]^{\mathbf{2 0}_{\mathbf{D}}}=+166.5(c=1.19, \mathrm{MeOH}) . \quad{ }^{1} \mathbf{H} \mathbf{~ N M R}$ $\left(\mathrm{CD}_{3} \mathrm{OD}, 400 \mathrm{MHz}\right) \delta 0.75(\mathrm{t}, 3 \mathrm{H}, J=7.5 \mathrm{~Hz}), 1.33(\mathrm{~m}, 1 \mathrm{H}), 1.55(\mathrm{~m}, 1 \mathrm{H}), 3.52(\mathrm{~m}, 1 \mathrm{H}), 3.61(\mathrm{ddd}$, $1 \mathrm{H}, J=7.6,5.0,1.1 \mathrm{~Hz}), 3.87(\mathrm{dd}, 1 \mathrm{H}, J=11.2,7.6 \mathrm{~Hz}), 3.96(\mathrm{dd}, 1 \mathrm{H}, J=11.2,5.0 \mathrm{~Hz}), 4.25(\mathrm{~d}, 1$ $\mathrm{H}, J=14.2 \mathrm{~Hz}), 5.53(\mathrm{~d}, 1 \mathrm{H}, J=14.2 \mathrm{~Hz}), 7.57(\mathrm{~m}, 5 \mathrm{H}) .{ }^{1} \mathbf{H} \mathbf{N M R}\left(\right.$ DMSO-d $\left._{6}, 300 \mathrm{MHz}\right) \delta 0.49(\mathrm{t}$, $3 \mathrm{H}, J=7.3 \mathrm{~Hz}), 1.07(\mathrm{~m}, 1 \mathrm{H}), 1.27(\mathrm{~m}, 1 \mathrm{H}), 3.18(\mathrm{~m}, 1 \mathrm{H}), 3.45(\mathrm{~m}, 1 \mathrm{H}), 3.49(\mathrm{~m}, 1 \mathrm{H}), 3.59(\mathrm{~m}, 1$ H), $4.05(\mathrm{~d}, 1 \mathrm{H}, J=14.3 \mathrm{~Hz}), 5.10(\mathrm{~d}, 1 \mathrm{H}, J=14.3 \mathrm{~Hz}), 5.15(\mathrm{t}, 1 \mathrm{H}, J=5.2 \mathrm{~Hz}), 7.34(\mathrm{~m}, 5 \mathrm{H}), 8.65$ $(\mathrm{d}, 1 \mathrm{H}, J=4.9 \mathrm{~Hz}){ }^{13} \mathbf{C}$ NMR $\left(\right.$ DMSO-d $\left._{6}, 50 \mathrm{MHz}\right) \delta 10.4,28.2,49.2,50.6,58.0,61.0,128.5,129.3$ (2 C), 129.6 (2 C), 137.5, 157.4, 157.8. IR (film): $v=3413,2928,1665,1454,1117,1052,757,705$ $\mathrm{cm}^{-1}$. MS (ES): $297[\mathrm{M}+\mathrm{Cl}]^{+}$(100\%). Anal. calcd for $\mathrm{C}_{14} \mathrm{H}_{18} \mathrm{~N}_{2} \mathrm{O}_{3}$ (262.3): C, 64.10; H, 6.92; N, 10.68; found: C, 63.85; H, 7.04; N, 10.49. Data for 3a: $\boldsymbol{R}_{\mathbf{f}}=0.25\left(20: 1 \mathrm{CH}_{2} \mathrm{Cl}_{2}-\mathrm{EtOH}\right) . \quad[\alpha]^{20}{ }_{\mathbf{D}}=$ $+10.7(c=0.85) .{ }^{1} \mathbf{H}$ NMR $(400 \mathrm{MHz}) \delta 0.53(\mathrm{t}, 3 \mathrm{H}, J=7.3 \mathrm{~Hz}), 1.13(\mathrm{~m}, 1 \mathrm{H}, J=6.8 \mathrm{~Hz}), 1.37(\mathrm{~m}$, $1 \mathrm{H}, J=6.8 \mathrm{~Hz}), 2.42(\mathrm{~s}, 3 \mathrm{H}), 3.28(\mathrm{~m}, 1 \mathrm{H}), 3.47(\mathrm{dd}, 1 \mathrm{H}, J=8.7,4.3 \mathrm{~Hz}), 3.58(\mathrm{dd}, 1 \mathrm{H}, J=10.6$, $8.7 \mathrm{~Hz}), 3.81(\mathrm{~d}, 1 \mathrm{H}, J=14.2 \mathrm{~Hz}), 4.03(\mathrm{dd}, 1 \mathrm{H}, J=10.6,4.3 \mathrm{~Hz}), 5.27(\mathrm{~d}, 1 \mathrm{H}, J=14.2 \mathrm{~Hz}), 7.32$ $(\mathrm{m}, 5 \mathrm{H}), 7.34(\mathrm{~d}, 2 \mathrm{H}, J=8.2 \mathrm{~Hz}), 7.54(\mathrm{~d}, 2 \mathrm{H}, J=8.2 \mathrm{~Hz}) .{ }^{13} \mathbf{C} \mathbf{N M R}(50 \mathrm{MHz}) \delta 9.5,21.5,27.5$, 49.1, 51.4, 55.7, 60.6, 125.2 (2 C), 128.4, 128.9 (2 C), 129.3 (2 C), 130.1 (2 C), 135.3, 140.5, 143.6, 156.5, 157.6.

2.2. (+)-(5R,6S)-1-Benzyl-6-hydroxymethyl-5-(2-phenylethyl)piperazin-2,3-dione, 2b. From 1b (267 mg, $0.603 \mathrm{mmol})$ and diethyl oxalate $(0.47 \mathrm{~mL}, 3.618 \mathrm{mmol})$, following general procedure $\mathbf{B},(40$ h), a mixture of diketopiperazine $\mathbf{2 b}$ and morpholinedione $\mathbf{3 b}$ was obtained. Purification by 
chromatography afforded $96 \mathrm{mg}(0.284 \mathrm{mmol}, 47 \%)$ of $\mathbf{2 b}$ as a white foam, and $140 \mathrm{mg}(0.282 \mathrm{mmol}$, $47 \%)$ of $\mathbf{3 b}$ as a white foam. Subsequently, a solution of $\mathrm{NaOMe}$ in $\mathrm{MeOH}(0.24 \mathrm{M}, 0.60 \mathrm{~mL}, 0.14$ mmol) was added to a solution of $\mathbf{3 b}$ in methanol $(10 \mathrm{~mL} / \mathrm{mmol})$ following the general procedure $(1 \mathrm{~h}$ $30 \mathrm{~min})$. After purification by chromatography $\left(50 \% \mathrm{Et}_{2} \mathrm{O}-\mathrm{CH}_{2} \mathrm{Cl}_{2}\right.$, then $\left.2-10 \% \mathrm{MeOH}-\mathrm{CH}_{2} \mathrm{Cl}_{2}\right) 64 \mathrm{mg}$ of $\mathbf{2} \mathbf{b}$ was obtained $(0.189 \mathrm{mmol}, 31 \%)$ as a white foam which was recrystallized from $\mathrm{Et}_{2} \mathrm{O}$. The global yield was 78\%. Data for $\mathbf{2 b}$ : $\mathbf{m p}: 150-152{ }^{\circ} \mathrm{C}\left(\mathrm{Et}_{2} \mathrm{O}\right) . \quad \boldsymbol{R}_{\mathbf{f}}=0.23\left(10 \% \mathrm{MeOH}-\mathrm{CH}_{2} \mathrm{Cl}_{2}\right) . \quad[\alpha]^{\mathbf{2 0}}{ }_{\mathbf{D}}=$ $+177.8(c=1.11) .{ }^{1} \mathbf{H}$ NMR $(300 \mathrm{MHz}) \delta 1.41(\mathrm{~m}, 1 \mathrm{H}), 1.64(\mathrm{~m}, 1 \mathrm{H}), 2.07(\mathrm{~m}, 1 \mathrm{H}), 2.24(\mathrm{~m}, 1 \mathrm{H})$, $3.37($ ap t, $1 \mathrm{H}, J=5.6 \mathrm{~Hz}), 3.56(\mathrm{~m}, 1 \mathrm{H}), 3.77$ (AB system, $2 \mathrm{H}), 3.94(\mathrm{~d}, 1 \mathrm{H}, J=14.2 \mathrm{~Hz}), 5.34(\mathrm{~d}, 1$ $\mathrm{H}, J=14.3 \mathrm{~Hz}), 6.81(\mathrm{~d}, 2 \mathrm{H}, J=6.5 \mathrm{~Hz}), 7.10(\mathrm{~m}, 3 \mathrm{H}), 7.29(\mathrm{~m}, 5 \mathrm{H}), 8.29(\mathrm{~d}, 1 \mathrm{H}, J=5.2 \mathrm{~Hz}) .{ }^{13} \mathrm{C}$ NMR (50 MHz) $\delta \quad 31.4,35.9,49.2,49.8,57.1,61.1,125.8$ (2 C), 128.1 (2 C), $128.2(2 \mathrm{C}), 128.5(2$ C), 128.9 (2 C), 135.6, 140.3, 157.9, 158.4. IR (KBr): $v=3435,2920,2855,1714,1666,1453,700$ $\mathrm{cm}^{-1}$. MS (ES): $699[2 \mathrm{M}+\mathrm{Na}]^{+}, 361[\mathrm{M}+\mathrm{Na}]^{+}, 339[\mathrm{M}+1]^{+}(100 \%)$. Anal. calcd for $\mathrm{C}_{20} \mathrm{H}_{22} \mathrm{~N}_{2} \mathrm{O}_{3} \mathrm{~S}$ (338.2): C, 70.97; H, 6.56; N, 8.28; found: C, 70.85; H, 6.80; N, 8.22. Partial data for 3b: $\boldsymbol{R}_{\mathbf{f}}=0.40$ $\left(5 \% \mathrm{MeOH}-\mathrm{CH}_{2} \mathrm{Cl}_{2}\right) .{ }^{1} \mathrm{H}$ NMR $(200 \mathrm{MHz}) \delta 1.44(\mathrm{~m}, 1 \mathrm{H}), 1.65(\mathrm{~m}, 1 \mathrm{H}), 2.16(\mathrm{t}, 2 \mathrm{H}, J=7.7 \mathrm{~Hz})$, $2.41(\mathrm{~s}, 3 \mathrm{H}), 3.38(\mathrm{~m}, 1 \mathrm{H}), 3.53(\mathrm{~m}, 3 \mathrm{H}), 3.82(\mathrm{~d}, 1 \mathrm{H}, J=14.1 \mathrm{~Hz}), 3.97(\mathrm{~m}, 2 \mathrm{H}), 5.28(\mathrm{~d}, 1 \mathrm{H}, J=$ $14.1 \mathrm{~Hz}), 6.86(\mathrm{dd}, 2 \mathrm{H}, J=7.9,2.0 \mathrm{~Hz}), 7.10-7.34(\mathrm{~m}, 10 \mathrm{H}), 7.50(\mathrm{~d}, 2 \mathrm{H}, J=8.2 \mathrm{~Hz})$.

2.3. (+)-(5R,6S)-1-Benzyl-6-hydroxymethyl-5-(i-propyl)piperazin-2,3-dione, 2c. From 1c (250 mg, $0.69 \mathrm{mmol})$ and diethyl oxalate $(0.56 \mathrm{~mL}, 4.14 \mathrm{mmol})$, following general procedure B $(48 \mathrm{~h})$, diketopiperazine $2 \mathbf{c}$ was obtained as a white solid by washing the crude product with $\mathrm{Et}_{2} \mathrm{O}$. The mother liquors containing morpholinedione $3 \mathbf{c}$ were concentrated under reduced pressure and redisolved in methanol $(10 \mathrm{~mL} / \mathrm{mmol})$. To this mixture a solution of $\mathrm{NaOMe}$ in $\mathrm{MeOH}(0.16 \mathrm{M}, 2.00 \mathrm{~mL}, 0.32$ mmol) was added. According to the general procedure (72 h) diketopiperazine $\mathbf{2 c}$ was obtained. Finally,

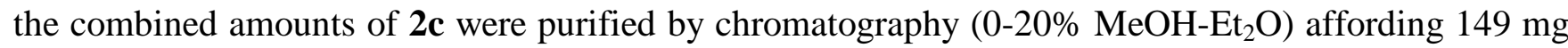
$(0.53 \mathrm{mmol}, 77 \%)$ of $\mathbf{2 c}$ as a white solid. $\quad$ Data for $\mathbf{2 c}: \mathbf{m p}: 184-186{ }^{\circ} \mathrm{C} . \quad \boldsymbol{R}_{\mathbf{f}}=0.34(10 \% \mathrm{MeOH}-$ $\left.\mathrm{Et}_{2} \mathrm{O}\right) .[\alpha]^{20}{ }_{\mathrm{D}}=+167.6(c=0.52) .{ }^{1} \mathbf{H} \mathbf{N M R}(300 \mathrm{MHz}) \delta 0.31(\mathrm{~d}, 3 \mathrm{H}, J=6.5 \mathrm{~Hz}), 0.84(\mathrm{~d}, 3 \mathrm{H}, J=$ 
$6.7 \mathrm{~Hz}), 1.44(\mathrm{~m}, 1 \mathrm{H}), 3.06(\mathrm{dd}, 1 \mathrm{H}, J=9.5,5.5 \mathrm{~Hz}), 3.48($ ap t, $1 \mathrm{H}, J=5.8 \mathrm{~Hz}), 3.79(\mathrm{~d}, 2 \mathrm{H}, J=5.7$ Hz), 3.97 (d, $1 \mathrm{H}, J=14.2 \mathrm{~Hz}), 4.45$ (br s, $1 \mathrm{H}), 5.53$ (d, $1 \mathrm{H}, J=14.3 \mathrm{~Hz}), 7.31$ (m, $5 \mathrm{H}), 8.09$ (d, $1 \mathrm{H}$, $J=5.0 \mathrm{~Hz}) .{ }^{13} \mathbf{C}$ NMR $(50 \mathrm{MHz}) \delta 18.5,19.0,31.6,49.2,55.7,56.1,61.4,128.3,128.8(2 \mathrm{C}), 129.4$ (2 C), 135.7, 157.8, 158.4. IR (KBr): $v=3433,2963,2927,1703,1661,1450,1059,702 \mathrm{~cm}^{-1}$. MS (ES): $575[2 \mathrm{M}+\mathrm{Na}]^{+}, 553[2 \mathrm{M}+1]^{+}, 277[\mathrm{M}+1]^{+}(100 \%)$. Anal. calcd for $\mathrm{C}_{15} \mathrm{H}_{20} \mathrm{~N}_{2} \mathrm{O}_{3}(276.3)$ : C, 65.20; H, 7.30; N, 10.14; found: C, 65.03; H, 7.38; N, 10.27. Partial data for $3 \mathbf{c}$ (from the crude): $\boldsymbol{R}_{\mathbf{f}}=$ $0.50\left(10 \% \mathrm{MeOH}_{\left.-\mathrm{Et}_{2} \mathrm{O}\right) .}{ }^{1} \mathbf{H}\right.$ NMR $(200 \mathrm{MHz}) \delta 2.38(\mathrm{~s}, 3 \mathrm{H}), 5.29(\mathrm{~d}, 1 \mathrm{H}, J=14.1 \mathrm{~Hz})$.

2.4. (+)-(5R,6S)-1-Benzyl-6-hydroxymethyl-5-phenylpiperazin-2,3-dione, 2d. Diketopiperazine 2d was obtained from $1 \mathbf{d}(100 \mathrm{mg}, 0.25 \mathrm{mmol})$, diethyl oxalate $(0,20 \mathrm{~mL}, 1.52 \mathrm{mmol})$ and 2 equiv of a $\mathrm{NaOMe}$ in $\mathrm{MeOH}(0.25 \mathrm{M}, 2 \mathrm{~mL}, 0.50 \mathrm{mmol})$ following general procedure A. Purification by washing thoroughly the crude product with $\mathrm{CH}_{2} \mathrm{Cl}_{2}$ afforded $70 \mathrm{mg}(0.23 \mathrm{mmol}, 90 \%)$ of $\mathbf{2 d}$ as a white solid. Data for 2d: $\mathbf{m p}: 190-195{ }^{\circ} \mathrm{C} . \quad \boldsymbol{R}_{\mathbf{f}}=0.15\left(10: 1 \mathrm{CH}_{2} \mathrm{Cl}_{2}-\mathrm{MeOH}\right) . \quad[\alpha]^{\mathbf{2 0}}=+47.7(c=0.66, \mathrm{MeOH}) .{ }^{\mathbf{1}} \mathbf{H}$ NMR (DMSO-d 6 , 300 MHz) $\delta 3.48(\mathrm{~m}, 1 \mathrm{H}), 3.63(\mathrm{~m}, 1 \mathrm{H}), 3.75(\mathrm{~m}, 1 \mathrm{H}), 4.00(\mathrm{~d}, 1 \mathrm{H}, J=14.9 \mathrm{~Hz})$, $4.72(\mathrm{~d}, 1 \mathrm{H}, J=5.2 \mathrm{~Hz}), 4.87(\mathrm{~d}, 1 \mathrm{H}, J=14.9 \mathrm{~Hz}), 5.35(\mathrm{t}, 1 \mathrm{H}, J=5.2 \mathrm{~Hz}), 6.67(\mathrm{~d}, 2 \mathrm{H}, J=7.2 \mathrm{~Hz})$ $6.96(\operatorname{ap~t}, 2 \mathrm{H}, J=7.2 \mathrm{~Hz}), 7.04(\mathrm{~m}, 6 \mathrm{H}), 8.92(\mathrm{~d}, 1 \mathrm{H}, J=4.5 \mathrm{~Hz}) .{ }^{13} \mathbf{C}$ NMR (DMSO-d $\left.6,50 \mathrm{MHz}\right) \delta$ 48.8, 52.1, 61.1, 62.7, 125.9 (2 C), 127.2, 127.7, 127.8 (2 C), 128.3 (2 C), 128.7 (2 C), 136.1, 140.9, 157.6, 157.9. IR (KBr): $v=3430,1638,1630,1454,1175,1100,1000,690 \mathrm{~cm}^{-1} . \quad$ MS (ES): 333 $[\mathrm{M}+\mathrm{Na}]^{+}(100 \%)$. Anal. calcd for $\mathrm{C}_{18} \mathrm{H}_{18} \mathrm{~N}_{2} \mathrm{O}_{3}$ (310.3): C, 69.66; H, 5.85; N, 9.03; found: C, 69.90; H, 5.53; N, 9.12.

2.5. (+)-(5R,6S)-1-Benzyl-5-(p-fluorophenyl)-6-hydroxymethylpiperazin-2,3-dione, 2 e.

Diketopiperazine 2e was obtained from 1e (140 mg, $0.34 \mathrm{mmol})$, diethyl oxalate $(0.28 \mathrm{~mL}, 2.03 \mathrm{mmol})$ and 2 equiv of a solution of $\mathrm{NaOMe}$ in $\mathrm{MeOH}(0.25 \mathrm{M}, 2.70 \mathrm{~mL}, 0.68 \mathrm{mmol})$, following general procedure A. Purification by chromatography (0-10\% $\left.\mathrm{MeOH}-\mathrm{CH}_{2} \mathrm{Cl}_{2}\right)$ afforded $91 \mathrm{mg}(0.28 \mathrm{mmol}$, $82 \%)$ of $2 \mathbf{e}$ as a white solid. Data of $\mathbf{2 e}: \mathbf{m p}: 237-240{ }^{\circ} \mathrm{C} . \quad \boldsymbol{R}_{\mathbf{f}}=0.17\left(10 \% \mathrm{MeOH}-\mathrm{CH}_{2} \mathrm{Cl}_{2}\right) .[\alpha]^{20}{ }_{\mathbf{D}}=$ +49.0 (c= 1.10, DMSO). ${ }^{1}$ H NMR $\left(\right.$ DMSO-d $_{6}, 300$ MHz) $\delta 3.42($ ap t, 1 H), 3.63 (ap t, 1 H), $3.74(\mathrm{dd}$ 
$1 \mathrm{H}, J=10.8,4.8 \mathrm{~Hz}), 3.91(\mathrm{~d}, 1 \mathrm{H}, J=14.6 \mathrm{~Hz}), 4.68($ ap s, $1 \mathrm{H}), 4.94(\mathrm{~d}, 1 \mathrm{H}, J=14.6 \mathrm{~Hz}), 5.36$ (br s, $1 \mathrm{H}), 6.72(\mathrm{~d}, 2 \mathrm{H}, J=7.2 \mathrm{~Hz}), 6.95-7.11(\mathrm{~m}, 7 \mathrm{H}), 8.94(\mathrm{~d}, 1 \mathrm{H}, J=4.9 \mathrm{~Hz}) .{ }^{13} \mathbf{C}$ NMR $\left(\mathrm{DMSO}_{6} \mathrm{~d}_{6}\right.$ $50 \mathrm{MHz}) \delta 48.3,51.3,60.8,62.1,115.1\left(\mathrm{~d}, 2 \mathrm{C}, J_{O} \mathrm{C}-\mathrm{F}=21.7 \mathrm{~Hz}\right), 126.9,127.4\left(\mathrm{~d}, 2 \mathrm{C}, J_{m} \mathrm{C}-\mathrm{F}=8.7\right.$ Hz), 128.7 (2 C), 127.9 (2 C), 135.7, 136.6, 157.2, 157.6, 161.5 (d, 1 C, Jipso C-F = 240.1 Hz). IR $(\mathrm{KBr}): v=3392,3239,2927,2507,1700,1668,1509,1450,1237,729 \mathrm{~cm}^{-1} . \quad$ MS (ES): 680 $[2 \mathrm{M}+\mathrm{Na}+1]^{+}, 679[2 \mathrm{M}+\mathrm{Na}]^{+}, 329[\mathrm{M}+1]^{+}(100 \%) .{ }^{1} \mathrm{H}$ and ${ }^{13} \mathrm{C} \mathrm{NMR}$ spectral assignments were supported by a 2D NMR technique (HMQC).

2.6 . (-)-(5R,6S)-1-Benzyl-6-hydroxymethyl-5-(1-naphtyl)piperazine-2,3-dione, 2f.

Diketopiperazine $2 \mathbf{f}$ was obtained from $\mathbf{1 f}(79 \mathrm{mg}, 0.178 \mathrm{mmol})$, diethyl oxalate $(0.14 \mathrm{~mL}, 1.068 \mathrm{mmol})$ and 0.5 equiv of a solution $0.25 \mathrm{M}$ of $\mathrm{NaOMe}$ in $\mathrm{MeOH}(1.42 \mathrm{~mL}, 0.356 \mathrm{mmol})$ following general procedure A. After recrystallization $\left(\mathrm{CH}_{2} \mathrm{Cl}_{2}\right)$ 2f was obtained as a white solid (46 $\mathrm{mg}, 0.134 \mathrm{mmol}$, $75 \%)$. Data for $\mathbf{2 f :} \quad \boldsymbol{R}_{\mathbf{f}}=0.15\left(30 \% \mathrm{MeOH}-\mathrm{CH}_{2} \mathrm{Cl}_{2}\right) . \quad \mathbf{m p}: 270-273{ }^{\circ} \mathrm{C} . \quad[\alpha]^{\mathbf{2 0}}=-16.7(c=0.51$, DMSO). ${ }^{1} \mathbf{H}$ NMR (DMSO-d $\left.6,300 \mathrm{MHz}\right) \delta 3.61(\mathrm{~m}, 1 \mathrm{H}), 3.73-3.87$ (m, $\left.2 \mathrm{H}\right), 3.87$ (d, $1 \mathrm{H}, J=14.9$ $\mathrm{Hz}), 4.84(\mathrm{~d}, 1 \mathrm{H}, J=14.9 \mathrm{~Hz}), 5.57(\mathrm{~d}, 1 \mathrm{H}, J=4.9 \mathrm{~Hz}), 5.73(\mathrm{t}, 1 \mathrm{H}, J=6.0 \mathrm{~Hz}), 6.41(\mathrm{~d}, 2 \mathrm{H}, J=7.2$ Hz), $6.65(\mathrm{t}, 2 \mathrm{H}, J=7.4 \mathrm{~Hz}), 6.78(\mathrm{t}, 1 \mathrm{H}, J=7.3 \mathrm{~Hz}), 7.19(\mathrm{~d}, 1 \mathrm{H}, J=7.2 \mathrm{~Hz}), 7.46-7.51(\mathrm{~m}, 3 \mathrm{H})$, 7.85-7.93 (d, $3 \mathrm{H}), 8.99(\mathrm{~d}, 1 \mathrm{H}, J=4.1 \mathrm{~Hz}) .{ }^{13} \mathrm{C}$ NMR (DMSO-d 6 , $\left.75 \mathrm{MHz}\right) \delta$ 48.0, 48.8, 60.1, 60.5, $121.9,123.6,124.9,125.7,126.4,126.6,127.0$ (2 C), 127.5 (2 C), 128.4, 128.9, 129.1, 133.7, 135.0, 135.3, 157.0, 158.0. IR (KBr): $v=3435,2920,2840,2536,1696,1667,1655,1447,1223,1115,771$, $699 \mathrm{~cm}^{-1}$. MS (ES): $743[2 \mathrm{M}+\mathrm{Na}]^{+}, 383[\mathrm{M}+\mathrm{Na}]^{+}(100 \%), 361[\mathrm{M}+1]^{+}$. Anal. calcd for $\mathbf{C}_{\mathbf{2 2}} \mathbf{H}_{\mathbf{2 0}} \mathbf{N}_{\mathbf{2}} \mathbf{O}_{\mathbf{3}}$ (360.4): C, 73.32; H, 5.59; N, 7.77; found: C, 73.17; H, 5.51; N, 7.92.

3. General Procedure of Reduction with $\mathbf{B H}_{3} \cdot \mathbf{S M e}_{2}$. To a solution of diketopiperazine in dry THF $(10 \mathrm{~mL} / \mathrm{mmol})$ under reflux was added dropwise 9 equiv of a $2 \mathrm{M}$ solution of $\mathrm{BH}_{3} \cdot \mathrm{SMe}_{2}$ in THF. The mixture was refluxed for $7 \mathrm{~h}$, the solvent was evaporated under reduced pressure and 4 equiv of a 0.2 $0.4 \mathrm{M} \mathrm{HCl}$ solution was added. The mixture was stirred for $30 \mathrm{~min}$ at $100{ }^{\circ} \mathrm{C}$ and then it was cooled at 
$0{ }^{\circ} \mathrm{C}$ and 6 equiv of a $0.2-0.4 \mathrm{M}$ solution of $\mathrm{NaOH}$ was added and was stirred for $1 \mathrm{~h}$ and $30 \mathrm{~min}$. The aqueous mixture was saturated with solid $\mathrm{K}_{2} \mathrm{CO}_{3}$, extracted with $\mathrm{CH}_{2} \mathrm{Cl}_{2}$ (3-4 times, $5 \mathrm{~mL} / \mathrm{mmol}$ ) and the combined organic extracts were dried over $\mathrm{Na}_{2} \mathrm{SO}_{4}$ and filtered to give, after evaporation of the solvent, a crude product that was purified by chromatography on silica gel using the appropriate mixture of solvents.

3.1. (-)-(2S,3R)-1-Benzyl-3-ethyl-2-hydroxymethylpiperazine, 4a. Piperazine 4a

(123 $\mathrm{mg}$, $0.525 \mathrm{mmol}, 75 \%)$ was obtained as a colorless oil from $\mathbf{2 a}(188 \mathrm{mg}, 0.713 \mathrm{mmol})$ and $\mathrm{BH}_{3} \cdot \mathrm{SMe}_{2}(2 \mathrm{M}$, $3.21 \mathrm{~mL}, 6.417 \mathrm{mmol})$ following the general procedure $(7 \mathrm{~h})$ and after purification by chromatography $\left(0-20 \% \mathrm{MeOH}-\mathrm{Et}_{2} \mathrm{O}\right)$. Data for 4a: $\quad \boldsymbol{R}_{\mathbf{f}}=0.10\left(40 \% \mathrm{MeOH}_{-} \mathrm{Et}_{2} \mathrm{O}\right) . \quad[\alpha]^{\mathbf{2 0}}{ }_{\mathbf{D}}=-14.1(c=0.91) . \quad{ }^{\mathbf{1}} \mathbf{H}$ NMR (300 MHz) $\delta 0.92(\mathrm{t}, 3 \mathrm{H}, J=7.3 \mathrm{~Hz}), 1.48(\mathrm{sept}, 1 \mathrm{H}, J=7.6 \mathrm{~Hz}), 1.72(\mathrm{~m}, 1 \mathrm{H}), 2.19(\mathrm{~m}, 1 \mathrm{H})$, $2.29(\mathrm{dt}, 1 \mathrm{H}, J=9.9,3.0 \mathrm{~Hz}), 2.70(\mathrm{~m}, 1 \mathrm{H}), 2.78-2.96(\mathrm{~m}, 3 \mathrm{H}), 3.29(\mathrm{~d}, 1 \mathrm{H}, J=13.3 \mathrm{~Hz}), 3.65$ (dd, $1 \mathrm{H}, J=11.7,1.6 \mathrm{~Hz}), 4.02(\mathrm{~d}, 1 \mathrm{H}, J=13.3 \mathrm{~Hz}), 4.05(\mathrm{dd}, 1 \mathrm{H}, J=11.8,3.2 \mathrm{~Hz}), 7.26(\mathrm{~m}, 5 \mathrm{H}) .{ }^{13} \mathbf{C}$ NMR (50 MHz) $\delta 10.3,25.1,43.1,50.5,56.3,58.3,59.1,63.3,127.0,128.3$ (2 C), 128.7 (2 C), 138.4. IR (film): $v=3306,3057,3021,2958,1490,1452,1027,739,699 \mathrm{~cm}^{-1}$. MS (ES): $235[\mathrm{M}+1]^{+}$ (100\%). Anal. calcd for $\mathrm{C}_{14} \mathrm{H}_{22} \mathrm{~N}_{2} \mathrm{O}$ (234.2): C, 71.76; H, 9.46; N, 11.95; found: C, 71.85; H, 9.24; N, 11.69. ${ }^{1} \mathrm{H}$ and ${ }^{13} \mathrm{C}$ NMR spectral assignments were supported by $2 \mathrm{D}$ NMR techniques (COSY and HMQC) and DEPT.

3.2. (+)-(2S,3R)-1-Benzyl-2-hydroxymethyl-3-(2-phenylethyl)piperazine, $4 \mathrm{~b}$. Piperazine $4 \mathrm{~b}$ (51 mg, $0.164 \mathrm{mmol}, 59 \%)$ was obtained as a colorless oil from $\mathbf{2 b}(96 \mathrm{mg}, 0.284 \mathrm{mmol})$ and $\mathrm{BH}_{3} \cdot \mathrm{SMe}_{2}(2 \mathrm{M}$, $1.28 \mathrm{~mL}, 2.556 \mathrm{mmol})$ following the general procedure $(7 \mathrm{~h})$ and after purification by chromatography $\left(0-80 \% \mathrm{MeOH}-\mathrm{Et}_{2} \mathrm{O}\right)$. Data for $4 \mathbf{b}: \quad \boldsymbol{R}_{\mathbf{f}}=0.18\left(10 \% \mathrm{MeOH}-\mathrm{CH}_{2} \mathrm{Cl}_{2}\right) . \quad[\alpha]^{\mathbf{2 0}}{ }_{\mathbf{D}}=+9.8(c=1.40) . \quad{ }^{\mathbf{1}} \mathbf{H}$ NMR (300 MHz) $\delta$ 1.76-1.88 (m, 1 H), 2.01-2.11 (m, 1 H), 2.26 (m, 1 H), 2.34 (m, 1 H), 2.60 (ddd, 1 $\mathrm{H}, J=13.5,10.6,5.7 \mathrm{~Hz}), 2.75(\mathrm{~m}, 2 \mathrm{H}), 2.89-3.05(\mathrm{~m}, 5 \mathrm{H}), 3.36(\mathrm{~d}, 1 \mathrm{H}, J=13.4 \mathrm{~Hz}), 3.68(\mathrm{dd}, 1 \mathrm{H}$, $J=11.6,2.1 \mathrm{~Hz}), 4.00(\mathrm{~d}, 1 \mathrm{H}, J=13.4 \mathrm{~Hz}), 4.08(\mathrm{dd}, 1 \mathrm{H}, J=11.6,3.2 \mathrm{~Hz}), 7.14-7.34(\mathrm{~m}, 10 \mathrm{H}) .{ }^{13} \mathrm{C}$ NMR $(50 \mathrm{MHz}) \delta 32.6,34.0,42.5,49.8,54.7,58.4,59.0,62.3,126.0,127.2,128.3(2 \mathrm{C}), 128.4(2 \mathrm{C})$, 
128.5 (2 C), 128.8 (2 C), 138.2, 141.6. IR (film): $v=3311,3026,2925,1659,1494,1453,1028,744$, $698 \mathrm{~cm}^{-1}$. MS (ES): $311[\mathrm{M}+1]^{+}(100 \%)$. Anal. calcd for $\mathrm{C}_{20} \mathrm{H}_{26} \mathrm{~N}_{2} \mathrm{O}$ (310.4): C, 77.38; H, 8.44; N, 9.02; found: $\mathrm{C}, 71.20 ; \mathrm{H}, 8.20 ; \mathrm{N}, 9.33 .{ }^{1} \mathrm{H}$ and ${ }^{13} \mathrm{C}$ NMR spectral assignments were supported by 2D NMR techniques (COSY, HMQC and HMBC) and DEPT.

3.3. (-)-(2S,3R)-1-Benzyl-2-hydroxymethyl-3-i-propylpiperazine, 4c. From 2c (110 mg, 0.398 $\mathrm{mmol})$ and $\mathrm{BH}_{3} \cdot \mathrm{SMe}_{2}(2 \mathrm{M}, 1.79 \mathrm{~mL}, 3.582 \mathrm{mmol})$ following the general procedure $(7 \mathrm{~h})$ piperazine $\mathbf{4 c}$ was obtained. Purification by chromatography $\left(0-30 \% \mathrm{MeOH}-\mathrm{Et}_{2} \mathrm{O}\right)$ and a second chromatography $\left(30 \% \mathrm{MeOH}-\mathrm{CH}_{2} \mathrm{Cl}_{2}\right)$ afforded $(60 \mathrm{mg}, 0.242 \mathrm{mmol}, 61 \%)$ of $\mathbf{4 c}$ as a colorless oil. Data for $\mathbf{4 c}: \boldsymbol{R}_{\mathbf{f}}=$ $0.10\left(20 \% \mathrm{MeOH}-\mathrm{Et}_{2} \mathrm{O}\right) .[\alpha]^{\mathbf{2 0}}{ }_{\mathbf{D}}=-19.6(c=0.91) .{ }^{\mathbf{1}} \mathbf{H} \mathbf{N M R}(400 \mathrm{MHz}) \delta 0.87(\mathrm{~d}, 3 \mathrm{H}, J=1.8 \mathrm{~Hz})$, $0.89(\mathrm{~d}, 3 \mathrm{H}, J=1.8 \mathrm{~Hz}), 2.39(\mathrm{~m}, 2 \mathrm{H}), 2.48$ (ap quint, $1 \mathrm{H}, J=2.6 \mathrm{~Hz}$ ), 2.61 (dd, $1 \mathrm{H}, J=6.7,5.1$ Hz), $2.68(\mathrm{dd}, 1 \mathrm{H}, J=7.9,5.8 \mathrm{~Hz}), 2.98($ ap q, $2 \mathrm{H}, J=7.9 \mathrm{~Hz}), 3.04(\mathrm{br} \mathrm{s}, 2 \mathrm{H}), 3.48(\mathrm{~d}, 1 \mathrm{H}, J=13.3$ Hz), $3.66(\mathrm{dd}, 1 \mathrm{H}, J=11.5,2.6 \mathrm{~Hz}), 3.91(\mathrm{~d}, 1 \mathrm{H}, J=13.4 \mathrm{~Hz}), 4.15(\mathrm{dd}, 1 \mathrm{H}, J=11.6,2.7 \mathrm{~Hz}), 7.23$ (m, $1 \mathrm{H}), 7.29$ (m, $4 \mathrm{H}) .{ }^{13} \mathbf{C}$ NMR (50 MHz) $\delta 17.0,20.6,25.9,41.8,49.9,58.6,58.8,61.0,61.5$, 127.0, 128.3 (2 C), 128.7 (2 C), 138.9. IR (film): $v=3325,3028,2955,2869,1602,1452,1363$, 1071, 742, $698 \mathrm{~cm}^{-1}$. MS (ES): $249[\mathrm{M}+1]^{+}(100 \%)$. Anal. calcd for $\mathrm{C}_{15} \mathrm{H}_{24} \mathrm{~N}_{2} \mathrm{O}$ (248.4): C, 72.54; $\mathrm{H}$, 9.74; N, 11.28; found: $\mathrm{C}, 72.29 ; \mathrm{H}, 9.55 ; \mathrm{N}, 11.32 .{ }^{1} \mathrm{H}$ and ${ }^{13} \mathrm{C}$ NMR spectral assignments were supported by 2D NMR techniques (COSY, HMQC and HMBC) and DEPT.

3.4. (-)-(2S,3R)-1-Benzyl-2-hydroxymethyl-3-phenylpiperazine, 4d. Piperazine 4d (29 mg, 0.104 mmol, 69\%) was obtained as a colorless oil from $2 \mathbf{d}(47 \mathrm{mg}, 0.151 \mathrm{mmol})$ and $\mathrm{BH}_{3} \cdot \mathrm{SMe}_{2}(2 \mathrm{M}, 0.67$ $\mathrm{mL}, 1.359 \mathrm{mmol})$ following the general procedure $(8 \mathrm{~h})$ and after purification by chromatography $(0-$ $\left.20 \% \mathrm{MeOH}-\mathrm{Et}_{2} \mathrm{O}\right)$. Data for $4 \mathbf{d}: \quad \boldsymbol{R}_{\mathbf{f}}=0.26\left(25: 1 \mathrm{Et}_{2} \mathrm{O}-\mathrm{EtOH}\right) . \quad[\alpha]^{\mathbf{2 0}}{ }_{\mathbf{D}}=-67.2(c=0.91) .{ }^{\mathbf{1}} \mathbf{H} \mathbf{~ N M R}$ (300 MHz) $\delta 2.38($ br s, $2 \mathrm{H}), 2.44(\mathrm{~m}, 2 \mathrm{H}), 2.90(\mathrm{~m}, 3 \mathrm{H}), 3.22(\mathrm{~d}, 1 \mathrm{H}, J=13.3 \mathrm{~Hz}), 3.25(\mathrm{~d}, 1 \mathrm{H}, J=$ $11.8 \mathrm{~Hz}), 3.81(\mathrm{dd}, 1 \mathrm{H}, J=11.8,3.2 \mathrm{~Hz}), 3.93(\mathrm{~d}, 1 \mathrm{H}, J=9.5 \mathrm{~Hz}), 4.20(\mathrm{~d}, 1 \mathrm{H}, J=13.3 \mathrm{~Hz}), 7.24-$ 7.40 (m, 8 H), 7.43 (m, 2 H). ${ }^{13}$ C NMR (50 MHz) $\delta 45.8,52.0,58.0,58.1,61.9,66.7,127.2,127.9$, 
128.4 (4 C), 128.5 (2 C), 129.0 (2 C), 138.1, 142.0. IR (film): v = 3306, 3028, 2830, 1602, 1493, 1452, 1322, 1257, 1066, 1027, 910, 801, 759, 734, $700 \mathrm{~cm}^{-1}$. MS (ES): $283[\mathrm{M}+1]^{+}(100 \%)$.

\section{5. (-)-(2S,3R)-1-Benzyl-3-p-fluorophenyl-2-hydroxymethylpiperazine, 4e. Piperazine 4e was} obtained from $2 \mathrm{e}(65 \mathrm{mg}, 0.198 \mathrm{mmol})$ and $\mathrm{BH}_{3} \cdot \mathrm{SMe}_{2}(2 \mathrm{M}, 0.90 \mathrm{~mL}, 1.782 \mathrm{mmol})$ following the general procedure $(7 \mathrm{~h})$. Purification by chromatography $\left(0-20 \% \quad \mathrm{MeOH}_{-} \mathrm{Et}_{2} \mathrm{O}\right)$ followed by crystallization as hydrochloride [obtained by bubbling $\mathrm{HCl}(\mathrm{g})$ through an ethereal solution of $4 \mathbf{e}$ ] rendered pure $\mathbf{4 e}$ as a salt. Finally, standard basic treatment produced $\mathbf{4 e}, 50 \mathrm{mg}(0.166 \mathrm{mmol}, 84 \%)$, as a free base. Data for 4e: $\quad \boldsymbol{R}_{\mathbf{f}}=0.30\left(20 \% \mathrm{MeOH}-\mathrm{Et}_{2} \mathrm{O}\right) . \quad[\alpha]^{\mathbf{2 0}}=-50.5(c=0.40) .{ }_{\mathbf{D}}^{\mathbf{1}} \mathbf{H} \mathbf{~ N M R}(300$ MHz) $\delta 2.01($ br s, 2 H), 2.37-2.45 (m, 2 H), 2.84-2.90 (m, 3 H), $3.19(\mathrm{~d}, 1 \mathrm{H}, J=11.7 \mathrm{~Hz}), 3.21(\mathrm{~d}, 1$ $\mathrm{H}, J=13.4 \mathrm{~Hz}), 3.81(\mathrm{dd}, 1 \mathrm{H}, J=11.7,3.2 \mathrm{~Hz}), 3.93(\mathrm{~d}, 1 \mathrm{H}, J=9.5 \mathrm{~Hz}), 4.18(\mathrm{~d}, 1 \mathrm{H}, J=13.2 \mathrm{~Hz})$, $6.99($ ap t, $2 \mathrm{H}, J=8.7 \mathrm{~Hz}), 7.30(\mathrm{~m}, 5 \mathrm{H}), 7.37-7.42(\mathrm{~m}, 2 \mathrm{H}) .{ }^{13} \mathbf{C} \mathbf{N M R}(50 \mathrm{MHz}) \delta 45.8,52.0,58.0$ $(2 \mathrm{C}), 61.0,66.9,115.3\left(\mathrm{~d}, 2 \mathrm{C}, J_{O} \mathrm{C}-\mathrm{F}=21.0 \mathrm{~Hz}\right), 127.2,128.4(2 \mathrm{C}), 128.9(2 \mathrm{C}), 130.0\left(\mathrm{~d}, 2 \mathrm{C}, J_{m} \mathrm{C}-\right.$ $\mathrm{F}=8.0 \mathrm{~Hz}), 137.8,137.9\left(\mathrm{~d}, 1 \mathrm{C}, J_{p} \mathrm{C}-\mathrm{F}=3.4 \mathrm{~Hz}\right), 162.3\left(\mathrm{~d}, 1 \mathrm{C}, J_{i p s o} \mathrm{C}-\mathrm{F}=246.1 \mathrm{~Hz}\right) . \quad$ IR (film): $v$ $=3308,3028,2942,2832,1604,1510,1451,1223,1067,836,740,700 \mathrm{~cm}^{-1}$. MS (ES): $301[\mathrm{M}+1]^{+}$ $(100 \%)$.

\section{Synthesis of (-)-(2R,3S)-4-benzyl-1-benzyloxycarbonyl-2-ethyl-3-hydroxymethylpiperazine, 5.} To a solution of piperazine $4 \mathbf{a},(64 \mathrm{mg}, 0.273 \mathrm{mmol})$ in $\mathrm{CH}_{2} \mathrm{Cl}_{2}(6 \mathrm{~mL} / \mathrm{mmol}), 0.60 \mathrm{~mL}$ of a $1 \mathrm{~N}$ solution of $\mathrm{NaOH}(2 \mathrm{~mL} / \mathrm{mmol})$ and 0.9 equiv of a solution of $\mathrm{CbzCl}(0.2 \mathrm{M}, 35 \mu \mathrm{L}, 0.246 \mathrm{mmol})$ in $\mathrm{CH}_{2} \mathrm{Cl}_{2}$ were added at $0{ }^{\circ} \mathrm{C}$. The mixture was allowed to warm to room temperature $(2 \mathrm{~h})$ and then sequential additions of the $\mathrm{CbzCl}$ solution ( 3 times, 0.1 equiv) were carried out over a period of $5 \mathrm{~h}$, until disappearance of starting material (TLC). At that point $\mathrm{CH}_{2} \mathrm{Cl}_{2}$ was added and the layers were separated. The aqueous phase was extracted with $\mathrm{CH}_{2} \mathrm{Cl}_{2}(3$ times, $5 \mathrm{~mL} / \mathrm{mmol})$ and the combined organic extracts were washed with a saturated solution of $\mathrm{NaCl}(5 \mathrm{~mL} / \mathrm{mmol})$, dried over $\mathrm{Na}_{2} \mathrm{SO}_{4}$ and filtered to give, after evaporation of the solvents, a crude product that was purified by chromatography on silica gel (15-40\% EtOAc-hexane) affording $72 \mathrm{mg}(0.195 \mathrm{mmol}, 71 \%)$ of 5 as a colorless oil. Data 
for 5: This compound presents rotamers at room temperature therefore some signals are duplicated in the ${ }^{1} \mathrm{H}$ and ${ }^{13} \mathrm{C}$ NMR spectra. $\quad \boldsymbol{R}_{\mathbf{f}}=0.28(30 \%$ EtOAc-hexane $) . \quad[\alpha]^{\mathbf{2 0}}{ }_{\mathbf{D}}=-10.0(c=0.94) .{ }^{\mathbf{1}} \mathbf{H}$ NMR $\left(\mathrm{CD}_{3} \mathrm{OD}, 300 \mathrm{MHz}\right) \delta 1.01(\mathrm{~m}, 3 \mathrm{H}), 1.95(\mathrm{~m}, 1 \mathrm{H}), 2.18(\mathrm{~m}, 1 \mathrm{H}), 2.65(\mathrm{~m}, 1 \mathrm{H}), 2.80(\mathrm{~m}, 1 \mathrm{H}), 2.88$ (m, $1 \mathrm{H}), 3.22-3.37(\mathrm{~m}, 1 \mathrm{H}), 3.78(\mathrm{~m}, 1 \mathrm{H}), 3.88(\mathrm{~d}, 1 \mathrm{H}, J=13.4 \mathrm{~Hz}), 4.03(\mathrm{~d}, 1 \mathrm{H}, J=13.4 \mathrm{~Hz}), 4.08$ $(\mathrm{m}, 2 \mathrm{H}), 4.41(\mathrm{~m}, 1 \mathrm{H}), 5.32(\mathrm{~m}, 2 \mathrm{H}), 7.38-7.55(\mathrm{~m}, 10 \mathrm{H}) .{ }^{13} \mathbf{C} \mathbf{N M R}(50 \mathrm{MHz}) \delta 10.6,22.9+23.4(1$ C), 37.3+38.5 (1 C), 44.8, 53.0+53.6 (1 C), 56.3+57.1 (1 C), 59.3, 60.3+60.8 (1 C), 67.1, 127.1, 127.7 (2 C), 128.0, 128.3 (2 C), 128.4 (2 C), 128.5 (2 C), 136.7, 139.1, 156.6. IR (film): v = 3437, 3063, 3030, 2961, 2869, 1694, 1495, 1427, 1355, 1328, 1280, 1229, 1133, 1102, 1048, 912, 738, $697 \mathrm{~cm}^{-1}$. MS (ES): $759[2 \mathrm{M}+\mathrm{Na}]^{+}, 369[\mathrm{M}+1]^{+}(100 \%)$, Anal. calcd for $\mathrm{C}_{22} \mathrm{H}_{28} \mathrm{~N}_{2} \mathrm{O}_{3}$ (368.5): C, 71.71; $\mathrm{H}$, 7.66; N, 7.60; found: $\mathrm{C}, 71.88 ; \mathrm{H}, 7.34 ; \mathrm{N}, 7.54 .{ }^{1} \mathrm{H}$ and ${ }^{13} \mathrm{C} \mathrm{NMR}$ spectral assignments were supported by 2D NMR techniques (COSY and HMQC).

5. General Procedure for the Synthesis of Chloroacetamides. To a cold $\left(0{ }^{\circ} \mathrm{C}\right)$ suspension of 6 or 10 in EtOAc $(10 \mathrm{~mL} / \mathrm{mmol})$ and a saturated solution of $\mathrm{NaHCO}_{3}(10 \mathrm{~mL} / \mathrm{mmol}), 1.2-2$ equiv of freshly distilled chloroacetyl chloride was added. The mixture was stirred and allowed to warm up to room temperature until disappearance of the starting material (TLC). The layers were separated and the aqueous phase was extracted twice with $\mathrm{CH}_{2} \mathrm{Cl}_{2}(5 \mathrm{~mL} / \mathrm{mmol})$. The combined organic extracts were washed with a saturated solution of $\mathrm{NaCl}$, dried over $\mathrm{Na}_{2} \mathrm{SO}_{4}$ and filtered to give, after evaporation of the solvents, a crude product that was purified by chromatography on silica gel to afford chloroacetamide $\mathbf{7}$ or $\mathbf{1 1 .}$

\section{1. (+)- $N$-Benzyl- $N$-[(1S,2R, $\left.S_{\mathrm{S}}\right)-1$-( $t$-butyldimethylsilyloxymethyl)-2-(p-tolylsulfinylamino)but-1-} yl] 2-chloroacetamide, 7a. From 6a $(91 \mathrm{mg}, 0.198 \mathrm{mmol})$ was obtained 7a $(87 \mathrm{mg}, 0.162 \mathrm{mmol}$, $82 \%$ ) as a colorless oil according to the general procedure and after purification by chromatography on silica gel $\left(0-20 \% \mathrm{Et}_{2} \mathrm{O}-\mathrm{CH}_{2} \mathrm{Cl}_{2}\right)$. Data for 7a: $\boldsymbol{R}_{\mathbf{f}}=0.50\left(30 \% \mathrm{Et}_{2} \mathrm{O}-\mathrm{CH}_{2} \mathrm{Cl}_{2}\right)$. $[\alpha]^{\mathbf{2 0}}{ }_{\mathbf{D}}=+51.8(c=$ 1.32). ${ }^{1}$ H NMR $(200 \mathrm{MHz}) \delta-0.45(\mathrm{~s}, 3 \mathrm{H}),-0.18(\mathrm{~s}, 3 \mathrm{H}), 0.70(\mathrm{~s}, 9 \mathrm{H}), 0.76-0.86(\mathrm{~m}, 3 \mathrm{H}), 1.16-$ 1.38 (m, $2 \mathrm{H}), 2.39$ (s, $3 \mathrm{H}), 3.66-3.87$ (m, $4 \mathrm{H}), 4.00(\mathrm{~d}, 1 \mathrm{H}, J=13.7 \mathrm{~Hz}), 4.28(\mathrm{~d}, 1 \mathrm{H}, J=13.9 \mathrm{~Hz})$, 
$4.62(\mathrm{~d}, 1 \mathrm{H}, J=8.8 \mathrm{~Hz}), 4.90(\mathrm{AB}$ system, $2 \mathrm{H}), 7.11-7.46(\mathrm{~m}, 7 \mathrm{H}), 7.57(\mathrm{~d}, 2 \mathrm{H}, J=8.2 \mathrm{~Hz}) .{ }^{{ }^{13} \mathrm{C}}$ NMR (50MHz) $\delta-6.4$ (2 C), 8.2, 17.8, 21.3, 25.3, 25.9 (3 C), 42.9, 49.4, 52.7, 58.5, 61.3, $124.9(2$ C), 125.6 (2 C), 127.3, 128.9 (2 C), 129.4 (2 C), 137.6, 141.2, 141.4, 169.8. IR (film): v = 3267, 2949, 2928, 2876, 2855, 1651, 1462, 1258, 1090, 1067, 835, 812, $755 \mathrm{~cm}^{-1} . \mathbf{M S}(\mathrm{ES}): 1097[2 \mathrm{M}+\mathrm{Na}]^{+}, 537$ $[\mathrm{M}+1]^{+}(100 \%)$

5.2. (+)- $N$-Benzyl- $N-\left[\left(1 S, 2 R, S_{\mathrm{S}}\right)-1-(t\right.$-butyldimethylsilyloxymethyl)-4-phenyl-2-(ptolylsulfinylamino)but-1-yl] 2-chloroacetamide, 7b. $7 \mathbf{b}$ (76 mg, $0.831 \mathrm{mmol}, 83 \%)$ was obtained as a white solid from $\mathbf{6 b}(80 \mathrm{mg}, 0.149 \mathrm{mmol})$ following the general procedure $(24 \mathrm{~h})$ and after purification by column chromatography (20-40\% Et $2 \mathrm{O}$-hexane) and crystallization $\left(\mathrm{CH}_{2} \mathrm{Cl}_{2}\right.$-hexane). Data for $7 \mathbf{b}$ : $\boldsymbol{R}_{\mathbf{f}}=0.18\left(60 \% \quad \mathrm{Et}_{2} \mathrm{O}-\right.$ hexane $) . \quad \mathbf{m p}: 110-112{ }^{\circ} \mathrm{C} . \quad[\alpha]^{\mathbf{2 0}}{ }_{\mathbf{D}}=+356.3(c=1.00) .{ }^{\mathbf{1}} \mathbf{H} \mathbf{~ N M R}(400 \mathrm{MHz})$ $\delta-0.44(\mathrm{~s}, 3 \mathrm{H}),-0.20(\mathrm{~s}, 3 \mathrm{H}), 0.71(\mathrm{~s}, 9 \mathrm{H}), 1.55(\mathrm{~m}, 2 \mathrm{H}), 2.35-2.41(\mathrm{~m}, 1 \mathrm{H}), 2.38(\mathrm{~s}, 3 \mathrm{H}), 2.46-$ $2.59(\mathrm{~m}, 1 \mathrm{H}), 3.71-3.87(\mathrm{~m}, 4 \mathrm{H}), 4.02(\mathrm{~d}, 1 \mathrm{H}, J=13.7 \mathrm{~Hz}), 4.27$ (d, $1 \mathrm{H}, J=13.9 \mathrm{~Hz}), 4.60$ (br s, 1 H), $4.85(\mathrm{~d}, 1 \mathrm{H}, J=17.0 \mathrm{~Hz}), 4.96(\mathrm{~d}, 1 \mathrm{H}, J=18.1 \mathrm{~Hz}), 7.02-7.08(\mathrm{~m}, 4 \mathrm{H})$, 7.14-7.34 (m, $8 \mathrm{H}), 7.60$ $(\mathrm{d}, 2 \mathrm{H}, J=8.1 \mathrm{~Hz}) .{ }^{13} \mathrm{C}$ NMR $(75 \mathrm{MHz}) \delta-6.4$ (2 C), 18.0, 21.4, 25.6 (3 C), 30.8, 35.0, 42.9, 49.4, 52.2, 58.7, 61.3, 125.6 (2 C), 125.8 (2 C), 127.4, 128.3 (3 C), 128.4 (2 C), 128.9 (2 C), 129.5 (2 C), 137.5, 141.5, 141.6, 141.9, 169.9. IR $(\mathrm{KBr}): v=3435,3028,2954,2928,2856,1634,1495,1452$, 1257, 1092, 1070, 986, 836, 813, 778, 727, $699 \mathrm{~cm}^{-1}$. MS (ES): $1249[2 \mathrm{M}+\mathrm{Na}]^{+}, 615[\mathrm{M}+3]^{+}, 613$ $[\mathrm{M}+1]^{+}(100 \%), 481[\mathrm{M}-(\mathrm{OTBDMS})]^{+}$. Anal. calcd for $\mathrm{C}_{33} \mathrm{H}_{45} \mathrm{ClN}_{2} \mathrm{O}_{3} \mathrm{SSi}$ (613.3): C, 64.62; H, 7.40; N, 4.57; found: C, 64.97; H, 7.77; N, 4.36.

5.3. (+)- $N$-Benzyl- $N-\left[\left(1 S, 2 R, S_{S}\right)-1-(t\right.$-butyldimethylsilyloxymethyl)-3-methyl-2-(ptolylsulfinylamino)but-1-yl]-2-chloroacetamide, 7c. 7c (53 mg, $0.096 \mathrm{mmol}, 75 \%)$ was obtained as a white foam from $\mathbf{6 c}(61 \mathrm{mg}, 0.129 \mathrm{mmol})$ following the general procedure $(22 \mathrm{~h})$ and after purification by column chromatography $\left(10-30 \% \mathrm{Et}_{2} \mathrm{O}\right.$-hexane $)$. Data for 7c: $\boldsymbol{R}_{\mathbf{f}}=0.28\left(60 \% \mathrm{Et}_{2} \mathrm{O}-\mathrm{hexane}\right) .[\alpha]^{\mathbf{2 0}}{ }_{\mathbf{D}}$ $=+56.7(c=2.40) .{ }^{1} \mathbf{H}$ NMR $(300 \mathrm{MHz})$ (some signals appear broadened and display fine splittings due to the presence of rotamers $) \delta-0.46+(-0.24)(2 \mathrm{~s}, 3 \mathrm{H}),-0.18+(-0.13)(2 \mathrm{~s}, 3 \mathrm{H}), 0.48(\mathrm{~d}, 3 \mathrm{H}, J=$ 
$6.8 \mathrm{~Hz}), 0.73$ (s, $9 \mathrm{H}), 0.81$ (d, $3 \mathrm{H}, J=7.8 \mathrm{~Hz}), 1.72$ (heptd, $1 \mathrm{H}, J=6.8,2.2 \mathrm{~Hz}), 2.38$ (s, $3 \mathrm{H}), 3.68$ $3.80(\mathrm{~m}, 4 \mathrm{H}), 3.99(\mathrm{~d}, 1 \mathrm{H}, J=13.9 \mathrm{~Hz}), 4.27(\mathrm{~d}, 1 \mathrm{H}, J=13.9 \mathrm{~Hz}), 4.40(\mathrm{~d}, 1 \mathrm{H}, J=9.3 \mathrm{~Hz}), 4.86(\mathrm{~d}$, $1 \mathrm{H}, J=18.6 \mathrm{~Hz}), 5.03(\mathrm{~d}, 1 \mathrm{H}, J=18.6 \mathrm{~Hz}), 7.18(\mathrm{~d}, 2 \mathrm{H}, J=6.8 \mathrm{~Hz}), 7.21-7.35(\mathrm{~m}, 5 \mathrm{H}), 7.57(\mathrm{~d}, 2$ $\mathrm{H}, J=8.3 \mathrm{~Hz}) .{ }^{13} \mathrm{C}$ NMR $(75 \mathrm{MHz}) \delta-6.5$ (2 C), 15.1, 17.8, 20.6, 21.4, 25.6 (3 C), 28.2, 43.3, 49.4, 56.1 (2 C), 61.7, 124.8 (2 C), 125.6 (2 C), 127.2, 128.9 (2 C), 129.4 (2 C), 137.5, 141.1, 141.4, 169.9. IR (film): v = 3293, 2952, 2927, 2855, 1654, 1495, 1463, 1256, 1090, 1070, 837, 811, 777, 728, 697 $\mathrm{cm}^{-1}$. MS (ES): $1124[2 \mathrm{M}+\mathrm{Na}]^{+}, 553[\mathrm{M}+3]^{+}, 551[\mathrm{M}+1]^{+}(100 \%), 475\left[\mathrm{M}-\left(\mathrm{COCH}_{2} \mathrm{Cl}\right)\right]^{+}$. Anal. calcd for $\mathrm{C}_{16} \mathrm{H}_{23} \mathrm{ClN}_{2} \mathrm{O}_{4} \mathrm{~S}$ (374.9): $\mathrm{C}, 51.26 ; \mathrm{H}, 6.18 ; \mathrm{Cl}, 9.46 ; \mathrm{N}, 7.47 ; \mathrm{O}, 17.07 ; \mathrm{S}, 8.55$; found: $\mathrm{C}$, 51.04; H, 6.11; N, 7.65.

5.4 . (+)- $N$-Benzyl- $N-\left[\left(1 S, 2 R, S_{S}\right)-1-(t\right.$-butyldimethylsilyloxymethyl)-2-(1-naphtyl)-2-(ptolylsulfinylamino)eth-1-yl]-2-chloroacetamide, 7d. 7d (443 mg, 0.699 mmol, 90\%) was obtained as a white solid from $\mathbf{6 d}(435 \mathrm{mg}, 0.780 \mathrm{mmol})$ following the general procedure $(6 \mathrm{~h})$ and after purification by column chromatography (10-20\% EtOAc-hexane). Data of 7d: $\boldsymbol{R}_{\mathbf{f}}=0.34(30 \%$ EtOAchexane). $\quad$ mp: $55-57{ }^{\circ} \mathrm{C} . \quad[\alpha]^{20}{ }_{\mathrm{D}}=+56.7(c=2.40) . \quad{ }^{\mathbf{1}} \mathbf{H}$ NMR $(400 \mathrm{MHz})$ (some signals appear broadened and display fine splittings due to the presence of rotamers $) \delta-0.88-(-0.06)(\mathrm{m}, 6 \mathrm{H}), 0.45-$ $0.86(\mathrm{~m}, 9 \mathrm{H}), 2.02-2.30(\mathrm{~m}, 3 \mathrm{H}), 3.00(\mathrm{~m}, 1 \mathrm{H}), 3.49(\mathrm{~m}, 1 \mathrm{H}), 4.05(\mathrm{br} \mathrm{s}, 1 \mathrm{H}), 4.07(\mathrm{~d}, 1 \mathrm{H}, J=15.0$ Hz), $4.46(\mathrm{~d}, 1 \mathrm{H}, J=14.1 \mathrm{~Hz}), 4.96(\mathrm{~m}, 1 \mathrm{H}), 5.01(\mathrm{~d}, 1 \mathrm{H}, J=18.7 \mathrm{~Hz}), 5.33(\mathrm{~d}, 1 \mathrm{H}, J=18.7 \mathrm{~Hz})$, $5.82(\mathrm{~m}, 1 \mathrm{H}), 6.30(\mathrm{~d}, 1 \mathrm{H}, J=6.3 \mathrm{~Hz}), 6.70-7.82(\mathrm{~m}, 14 \mathrm{H}), 8.61(\mathrm{~d}, 1 \mathrm{H}, J=8.2 \mathrm{~Hz}) .{ }^{13} \mathbf{C} \mathbf{N M R}(75$ $\mathrm{MHz}$ ) (some signals appear broadened and display fine splittings due to the presence of rotamers) $\delta-5.0,-4.9,18.2+18.7$ (1 C), 21.3+21.5 (1 C), 26.1 (3 C), 44.1, 48.5, 56.8, 58.1, 61.0, 124.8+125.1(1 C), 125.7 (2 C), 126.0, 126.3, 126.7 (2 C), 127.5, 128.0, 128.5+128.6 (1 C), 129.2 (2 C), 129.6, 129.8, $130.3,130.6,133.5,134.2,134.7,136.9,137.9,141.4+141.7(1 \mathrm{C}), 172.6 . \quad$ IR $(\mathrm{KBr}): v=3057,2949$, 2920, 2855, 1650, 1490, 1468, 1252, 1089, 836, $777 \mathrm{~cm}^{-1}$. MS (ES): $1123[2 \mathrm{M}+\mathrm{Na}]^{+}, 637[\mathrm{M}+3]^{+}$, 
$635[\mathrm{M}+1]^{+}(100 \%), 559\left[\mathrm{M}-\left(\mathrm{COCH}_{2} \mathrm{Cl}\right)+2\right]^{+}$. Anal. calcd for $\mathrm{C}_{35} \mathrm{H}_{43} \mathrm{ClN}_{2} \mathrm{O}_{3} \mathrm{SSi}$ (635.3): C, 66.17; H, 6.82; N, 4.41; found: C, 65.99; H, 7.00; N, 4.52 .

\section{5. (+)- $N$-Benzyl- $N$-[(1S,2R, $\left.S_{\mathrm{S}}\right)-1$-(hydroxymethyl)-2-(1-naphtyl)-2-(p-tolylsulfinylamino)eth-1-}

yl]-2-chloroacetamide, 7e. 7e (38 $\mathrm{mg}, 0.073 \mathrm{mmol}, 52 \%)$ was obtained as a white solid from 1 (6) $\mathrm{mg}, 0.140 \mathrm{mmol})$ following the general procedure (1 h $30 \mathrm{~min})$ and after purification by column chromatography (10-20\% EtOAc-hexane). Data for 7e: $\boldsymbol{R}_{\mathbf{f}}=0.30$ (30\% EtOAc-hexane). $\mathbf{m p}: 55-57{ }^{\circ} \mathrm{C}$. $[\alpha]^{20}{ }_{D}=+25.9(\mathrm{c}=0.52) . \quad{ }^{\mathbf{1}} \mathbf{H}$ NMR $(300 \mathrm{MHz})$ (some signals appear broadened and display fine splittings due to the presence of rotamers) $1.91(\mathrm{~s}, 3 \mathrm{H}), 2.40(\mathrm{~d}, 1 \mathrm{H}, J=16.8 \mathrm{~Hz}), 3.39($ ap td, $1 \mathrm{H}, J=$ 11.0, $4.0 \mathrm{~Hz}), 3.46(\mathrm{~m}, 1 \mathrm{H}), 3.86(\mathrm{~d}, 1 \mathrm{H}, J=16.3 \mathrm{~Hz}), 3.95(\mathrm{~s}, 2 \mathrm{H}), 4.64(\mathrm{~m}, 1 \mathrm{H}), 5.43(\mathrm{dd}, 1 \mathrm{H}, J=$ 7.3, 5.1 Hz), 5.52 (br s, $1 \mathrm{H}), 6.49(\mathrm{~d}, 2 \mathrm{H}, J=6.2 \mathrm{~Hz}), 6.67(\mathrm{~d}, 2 \mathrm{H}, J=7.9 \mathrm{~Hz}), 7.08(\mathrm{~m}, 3 \mathrm{H}), 7.24$ $(\mathrm{m}, 3 \mathrm{H}), 7.36(\mathrm{~d}, 2 \mathrm{H}, J=7.7 \mathrm{~Hz}), 7.45(\mathrm{ap} \mathrm{t}, 1 \mathrm{H}), 7.54(\mathrm{~d}, 1 \mathrm{H}, J=6.2 \mathrm{~Hz}), 7.71(\mathrm{~d}, 1 \mathrm{H}, J=8.1 \mathrm{~Hz})$, 7.76 (m, $1 \mathrm{H}), 8.12$ (br s, $1 \mathrm{H}) .{ }^{13} \mathbf{C}$ NMR (75 MHz) $\delta 20.8,42.2,49.3,55.2,58.5,66.6,121.2,125.4(2$ C), 125.6, 126.2 (3 C), 126.9 (2 C), 127.6, 128.2, 128.7, 128.8 (4 C), 129.2, 133.0, 133.8, 135.8, 137.9, 140.8, 169.3. IR (KBr): $v=3434,3057,2942,1643,1452,1061,779 \mathrm{~cm}^{-1}$. MS (ES): 523 $[\mathrm{M}+3]^{+}, 521[\mathrm{M}+1]^{+}(100 \%), 471\left[\mathrm{M}-\left(\mathrm{CH}_{2} \mathrm{Cl}\right)\right]^{+}, 445\left[\mathrm{M}-\left(\mathrm{COCH}_{2} \mathrm{Cl}\right)+1\right]^{+}$.

\section{6. (+)-Methyl} $\left[\left(2 S, 3 R, S_{S}\right)-2-c h l o r o a c e t y l a m i n o-3-(p-f l u o r o p h e n y l)-3-(p-\right.$ tolylsulfinylamino)]propanoate, 11a. 11a (125 mg, $0.293 \mathrm{mmol}, 90 \%)$ was obtained as a white foam from 10a (114 mg, $0.325 \mathrm{mmol})$ following the general procedure ( $2 \mathrm{~h} 15 \mathrm{~min}$ ) and was used without further purification. Data for 11a: $\boldsymbol{R}_{\mathbf{f}}=0.26\left(5 \% \mathrm{MeOH}-\mathrm{CH}_{2} \mathrm{Cl}_{2}\right) \cdot[\alpha]^{\mathbf{2 0}}{ }_{\mathbf{D}}=+2.6(c=1.40)$. ${ }^{\mathbf{1}} \mathbf{H} \mathbf{N M R}$ (200 MHz) $\delta 2.39$ (s, $3 \mathrm{H}), 3.49(\mathrm{~s}, 3 \mathrm{H}), 4.17$ (s, $2 \mathrm{H}), 4.51$ (ap t, $1 \mathrm{H}, J=8.1 \mathrm{~Hz}), 4.79(\mathrm{dd}, 1 \mathrm{H}, J=$ 8.6, 7.1 Hz), $5.76(\mathrm{~d}, 1 \mathrm{H}, J=7.7 \mathrm{~Hz}), 6.90-6.93(\mathrm{~m}, 4 \mathrm{H}), 7.18(\mathrm{~d}, 2 \mathrm{H}, J=8.2 \mathrm{~Hz}), 7.42(\mathrm{~d}, 2 \mathrm{H}, J=$ $8.2 \mathrm{~Hz}), 8.80(\mathrm{~d}, 1 \mathrm{H}, J=5.8 \mathrm{~Hz}) .{ }^{13} \mathbf{C} \mathbf{R M N}(50 \mathrm{MHz}) \delta 21.2,42.4,52.2,56.0,57.7,115.2\left(\mathrm{~d}, 2 \mathrm{C}, J_{o}\right.$ $\mathrm{C}-\mathrm{F}=21.7 \mathrm{~Hz}), 126.4(2 \mathrm{C}), 128.8\left(\mathrm{~d}, 2 \mathrm{C}, J_{m} \mathrm{C}-\mathrm{F}=8.0 \mathrm{~Hz}\right), 129.4(2 \mathrm{C}), 134.0\left(\mathrm{~d}, 1 \mathrm{C}, J_{p} \mathrm{C}-\mathrm{F}=3.0\right.$ Hz), 139.2, 141.5, 162.2 (d, 1 C, $J_{\text {ipso }}$ C-F = 247.2 Hz), 167.6, 170.0. IR (film): v = 3420, 3306, 3057, 
2952, 1745, 1672, 1648, 1606, 1535, 1513, 1437, 1227, 1162, 1086, 1050, 846, 814, 789, $732 \mathrm{~cm}^{-1}$. MS (ES): $875[2 \mathrm{M}+\mathrm{Na}]^{+}, 427[\mathrm{M}+1]^{+}(100 \%), 369[\mathrm{M}+\mathrm{Na}]^{+}$.

\section{7. ( \pm )-Methyl $\left[\left(2 S, 3 R, S_{\mathrm{S}}\right)-2\right.$-chloroacetylamino-4-methyl-3-( $p$-tolylsulfinylamino $\left.)\right]$ pentanoate,}

11b. 11b (115 mg, $0.307 \mathrm{mmol}, 95 \%)$ was obtained as a colorless oil from $10 b$ (96 mg, $0.322 \mathrm{mmol})$ following the general procedure (4 h $30 \mathrm{~min}$ ) and was used without further purification. Data for 11b: $\boldsymbol{R}_{\mathbf{f}}=0.39\left(5 \% \mathrm{MeOH}-\mathrm{CH}_{2} \mathrm{Cl}_{2}\right) .{ }^{1} \mathbf{H} \mathbf{N M R}(200 \mathrm{MHz}) \delta 0.83(\mathrm{~d}, 3 \mathrm{H}, J=6.8 \mathrm{~Hz}), 0.99(\mathrm{~d}, 3 \mathrm{H}, J=6.8$ Hz), $1.72(\mathrm{~m}, 1 \mathrm{H}), 2.39$ (s, $3 \mathrm{H}), 3.56$ (ap dt, $1 \mathrm{H}, J=7.5,4.0 \mathrm{~Hz}), 3.75$ (s, $3 \mathrm{H}), 4.07$ (s, $2 \mathrm{H}), 4.23$ (br m, $1 \mathrm{H}), 4.79(\mathrm{dd}, 1 \mathrm{H}, J=8.9,4.0 \mathrm{~Hz}), 7.28(\mathrm{~d}, 2 \mathrm{H}, J=8.3 \mathrm{~Hz}), 7.56(\mathrm{~d}, 2 \mathrm{H}, J=8.4 \mathrm{~Hz}), 7.80(\mathrm{~d}, 1$ $\mathrm{H}, J=8.8 \mathrm{~Hz}) .{ }^{13} \mathrm{C}$ NMR $(75 \mathrm{MHz}) \delta 18.6,19.8,21.3,29.6,42.6,52.7,54.1,61.1,125.6(2 \mathrm{C}), 129.6$ (2 C), 140.9, 141.9, 166.7, 171.2. IR (film): $v=3233,3056,2962,1748,1679,1535,1436,1354$, $1260,1215,1158,1087,1055,980,919,813,730 \mathrm{~cm}^{-1} . \mathbf{M S}(\mathrm{ES}): 771[2 \mathrm{M}+\mathrm{Na}]^{+}, 375[\mathrm{M}+1]^{+}$ $(100 \%), 219\left[\mathrm{M}-\left(\mathrm{TolSONH}_{2}+1\right)+1\right]^{+}$.

5.8. ( \pm$)$-Methyl $\left[\left(2 S, 3 R, S_{S}\right)\right.$-2-chloroacetylamino-3-(p-tolylsulfinylamino)]butanoate, 11c. 11c (42 $\mathrm{mg}, 0.133 \mathrm{mmol}, 66 \%)$ was obtained as a colorless oil from 10c (55 mg, $0.203 \mathrm{mmol})$ following the general procedure (3 h $15 \mathrm{~min}$ ) and after column purification $\left(0-5 \% \mathrm{MeOH}-\mathrm{CH}_{2} \mathrm{Cl}_{2}\right)$. Data for 11c: $\boldsymbol{R}_{\mathbf{f}}$ $=0.39\left(5 \% \mathrm{MeOH}-\mathrm{CH}_{2} \mathrm{Cl}_{2}\right) .{ }^{1} \mathbf{H}$ NMR $(300 \mathrm{MHz}) \delta 1.24(\mathrm{~d}, 3 \mathrm{H}, J=6.8 \mathrm{~Hz}), 2.42(\mathrm{~s}, 3 \mathrm{H}), 3.79(\mathrm{~s}, 3$ H), $3.97(\mathrm{~m}, 1 \mathrm{H}), 4.11(\mathrm{~s}, 2 \mathrm{H}), 4.31(\mathrm{~d}, 1 \mathrm{H}, J=7.3 \mathrm{~Hz}), 4.61(\mathrm{dd}, 1 \mathrm{H}, J=8.2,4.8 \mathrm{~Hz}), 7.31(\mathrm{~d}, 2 \mathrm{H}$, $J=8.3 \mathrm{~Hz}), 7.56(\mathrm{~d}, 2 \mathrm{H}, J=8.3 \mathrm{~Hz}), 7.71(\mathrm{~d}, 1 \mathrm{H}, J=7.6 \mathrm{~Hz}) .{ }^{13} \mathbf{C} \mathbf{N M R}(75 \mathrm{MHz}) \delta 19.4,21.3$, 42.5, 51.9, 52.8, 57.3, 125.7 (2 C), 129.7 (2 C), 141.0, 141.8, 166.9, 170.5. IR (film): $v=3233$, 3056, 2962, 1748, 1679, 1535, 1436, 1354, 1260, 1215, 1158, 1087, 1051, 812, $755 \mathrm{~cm}^{-1}$. MS (ES): $715[2 \mathrm{M}+\mathrm{Na}]^{+}, 369[\mathrm{M}+\mathrm{Na}]^{+}, 347[\mathrm{M}+1]^{+}(100 \%), 191\left[\mathrm{M}-\left(\mathrm{TolSONH} \mathrm{H}_{2}+1\right)+1\right]^{+}$.

6. General Procedure for the Synthesis of Ketopiperazines. A solution of chloroacetamide 7 or 11 in DMF $(10 \mathrm{~mL} / \mathrm{mmol})$ and 1.8 equiv of solid $\mathrm{Cs}_{2} \mathrm{CO}_{3}$ were stirred at $65^{\circ} \mathrm{C}$ until starting material disappearance monitored by TLC. The mixture was cooled down to room temperature and was diluted with $\mathrm{CH}_{2} \mathrm{Cl}_{2}(10 \mathrm{~mL} / \mathrm{mmol})$ and $\mathrm{H}_{2} \mathrm{O}(10 \mathrm{~mL} / \mathrm{mmol})$. The layers were separated and the organic phase 
was washed with cold water $(3 \times 10 \mathrm{~mL} / \mathrm{mmol})$ and a saturated solution of $\mathrm{NaHCO}_{3}(10 \mathrm{~mL} / \mathrm{mmol})$, dried over $\mathrm{Na}_{2} \mathrm{SO}_{4}$, filtered and concentrated under reduced pressure to give a crude product that was purified by gradient column chromatography.

6.1 . $(+)-\left(5 R, 6 S, S_{\mathrm{S}}\right)-1-B e n z y l-6-[(t-b u t y l d i m e t h y l s i l y l o x y)$ methyl]-5-ethyl-4- $(p-$ tolylsulfinyl)piperazin-2-one, 8a. 8a (269 $\mathrm{mg}, 0.54 \mathrm{mmol}, 92 \%)$ was obtained as a white foam following the general procedure $(2 \mathrm{~h})$ and after chromatography on silica gel $\left(0-30 \% \mathrm{Et}_{2} \mathrm{O}-\mathrm{CH}_{2} \mathrm{Cl}_{2}\right)$. Data for 8a: $\boldsymbol{R}_{\mathbf{f}}=0.27\left(10 \% \mathrm{Et}_{2} \mathrm{O}-\mathrm{CH}_{2} \mathrm{Cl}_{2}\right) .[\alpha]^{\mathbf{2 0}}{ }_{\mathbf{D}}=+61.1(c=1.10) .{ }^{\mathbf{1}} \mathbf{H} \mathbf{N M R}(500 \mathrm{MHz}) \delta 0.07(\mathrm{~s}$, $3 \mathrm{H}), 0.10(\mathrm{~s}, 3 \mathrm{H}), 0.54$ (t, $3 \mathrm{H}, J=7.5 \mathrm{~Hz}), 0.90(\mathrm{~s}, 9 \mathrm{H}), 1.33-1.42(\mathrm{~m}, 1 \mathrm{H}), 1.75-1.83(\mathrm{~m}, 1 \mathrm{H}), 2.39$ (s, $3 \mathrm{H}), 3.08(\mathrm{~d}, 1 \mathrm{H}, J=17.7 \mathrm{~Hz}), 3.26(\mathrm{ddd}, 1 \mathrm{H}, J=8.3,5.3,1.5 \mathrm{~Hz}), 3.66(\mathrm{dd}, 1 \mathrm{H}, J=9.8,8.5$ $\mathrm{Hz}), 3.71(\mathrm{dd}, 1 \mathrm{H}, J=9.7,5.3 \mathrm{~Hz}), 3.75(\mathrm{ddd}, 1 \mathrm{H}, J=10.2,4.7,1.5 \mathrm{~Hz}), 3.80(\mathrm{~d}, 1 \mathrm{H}, J=17.7 \mathrm{~Hz})$, $3.84(\mathrm{~d}, 1 \mathrm{H}, J=14.3 \mathrm{~Hz}), 5.33(\mathrm{~d}, 1 \mathrm{H}, J=14.3 \mathrm{~Hz}), 7.24-7.33(\mathrm{~m}, 7 \mathrm{H}), 7.47(\mathrm{~d}, 2 \mathrm{H}, J=8.1 \mathrm{~Hz})$. ${ }^{13}$ C NMR (50 MHz) $\delta-5.3,-5.2,10.3,18.1,21.3,22.5,25.8$ (3 C), 39.7, 48.6, 57.8, 59.1, 62.4, 125.6 (2 C), 128.0, 128.8 (2 C), 128.9 (2 C), 129.8 (2 C), 136.6, 139.3, 141.8, 165.5. IR (film): $v=2963$, 2855, 1732, 1646, 1435, 1260, 1090, 1018, $799 \mathrm{~cm}^{-1}$. MS (ES): $523[\mathrm{M}+\mathrm{Na}]^{+}, 503[\mathrm{M}+3]^{+}, 502$ $[\mathrm{M}+2]^{+}, 501[\mathrm{M}+1]^{+}(100 \%) .{ }^{1} \mathrm{H}$ and ${ }^{13} \mathrm{C}$ NMR spectral assignments were supported by $2 \mathrm{D}$ NMR techniques (COSY and HMQC).

6.2. $\quad(+)-\left(5 R, 6 S, S_{S}\right)-1-B e n z y l-6-[(t-$ butyldimethylsilyloxy $)$ methyl]-5-(2-phenylethyl)-4- $(p-$ tolylsulfinyl)piperazin-2-one, 8b. 8b (160 mg, $0.277 \mathrm{mmol}, 86 \%)$ was obtained from $7 \mathbf{b}(197 \mathrm{mg}$, $0.321 \mathrm{mmol})$ as a white solid following the general procedure $(3 \mathrm{~h} 30 \mathrm{~min})$ and after chromatography on silica gel $\left(20-40 \% \mathrm{Et}_{2} \mathrm{O}-\right.$ hexane $)$ and crystallization $\left(\mathrm{Et}_{2} \mathrm{O}\right)$. Data for $\mathbf{8 b}: \quad \boldsymbol{R}_{\mathbf{f}}=0.24\left(80 \% \mathrm{Et}_{2} \mathrm{O}-\right.$ hexane). $\mathbf{m p}: 126-128{ }^{\circ} \mathrm{C} .[\alpha]^{20}{ }_{\mathbf{D}}=+84.6(c=1.00) .{ }^{\mathbf{1}} \mathbf{H} \mathbf{N M R}(300 \mathrm{MHz}) \delta 0.06(\mathrm{~s}, 3 \mathrm{H}), 0.08(\mathrm{~s}, 3$ H), 0.89 (s, $9 \mathrm{H}), 1.59-1.72(\mathrm{~m}, 1 \mathrm{H}), 2.03-2.18(\mathrm{~m}, 1 \mathrm{H}), 2.21-2.23(\mathrm{~m}, 2 \mathrm{H}), 2.40$ (s, $3 \mathrm{H}), 3.12(\mathrm{~d}, 1$ $\mathrm{H}, J=17.8 \mathrm{~Hz}), 3.30(\mathrm{ddd}, 1 \mathrm{H}, J=8.3,5.5,1.5 \mathrm{~Hz}), 3.66(\mathrm{dd}, 1 \mathrm{H}, J=9.9,8.3 \mathrm{~Hz}), 3.69(\mathrm{dd}, 1 \mathrm{H}, J=$ 9.8, 5.6 Hz), $3.83(\mathrm{~d}, 1 \mathrm{H}, J=17.8 \mathrm{~Hz}), 3.87(\mathrm{~d}, 1 \mathrm{H}, J=14.2 \mathrm{~Hz}), 3.90(\mathrm{br} \mathrm{dd}, 1 \mathrm{H}, J=8.1,4.4 \mathrm{~Hz})$, $5.31(\mathrm{~d}, 1 \mathrm{H}, J=14.2 \mathrm{~Hz}), 6.92(\mathrm{~d}, 2 \mathrm{H}, J=6.8 \mathrm{~Hz}), 7.11-7.23(\mathrm{~m}, 3 \mathrm{H}), 7.27-7.34(\mathrm{~m}, 7 \mathrm{H}), 7.47(\mathrm{~d}, 2$ 
$\mathrm{H}, J=8.1 \mathrm{~Hz}) .{ }^{13} \mathrm{C}$ NMR $\delta-5.3$ (2 C), 18.2, 21.3, 25.8 (3 C), 30.8, 32.0, 39.9, 48.6, 56.5, 58.6, 62.4, 125.6 (2 C), 126.1, 128.2, 128.4 (4 C), 128.9 (2 C), 129.0 (2 C), 129.8 (2 C), 136.7, 139.3, 140.3, 141.9, 165.4. IR $(\mathrm{KBr}): v=3032,2950,2929,2856,1644,1478.1454,1431.1361,1254,1162$, 1108, 1089, 1067, 932, 841, 816, 774, 715, 656, 628, $499 \mathrm{~cm}^{-1}$. MS (ES): $577[\mathrm{M}+1]^{+}(100 \%)$. Anal. calcd (\%) for $\mathrm{C}_{33} \mathrm{H}_{44} \mathrm{~N}_{2} \mathrm{O}_{3} \mathrm{SSi}(576.9)$ : C, 68.71; H, 7.69; N, 4.86; S, 5.56; found: C, 68.46; H, $7.88 ; \mathrm{N}, 4.63 ; \mathrm{S}, 5.22$.

6.3. (+)-(5R,6S, $\left.S_{S}\right)-1-B e n z y l-6-[(t-$ butyldimethylsilyloxy $)$ methyl]-5-(i-propyl)-4-(ptolylsulfinyl)piperazin-2-one, 8c. 8c (38 mg, $0.074 \mathrm{mmol}, 77 \%)$ was obtained from 7c (53 mg, 0.096 $\mathrm{mmol}$ ) as a white foam following the general procedure (2 h $30 \mathrm{~min}$ ) and after chromatography on silica gel $\left(30-40 \% \mathrm{Et}_{2} \mathrm{O}-\right.$ hexane $)$. Data for 8c: $\quad \boldsymbol{R}_{\mathbf{f}}=0.21\left(80 \% \mathrm{Et}_{2} \mathrm{O}\right.$-hexane $) . \quad[\alpha]^{\mathbf{2 0}}=+6.2(c=1.40) .{ }^{\mathbf{1}} \mathbf{H}$ NMR $(300 \mathrm{MHz}) \delta 0.06(\mathrm{~s}, 3 \mathrm{H}), 0.10(\mathrm{~s}, 3 \mathrm{H}), 0.36(\mathrm{~d}, 3 \mathrm{H}, J=6.6 \mathrm{~Hz}), 0.91(\mathrm{~s}, 9 \mathrm{H}), 1.09$ (d, $3 \mathrm{H}, J$ $=6.6 \mathrm{~Hz}), 1.55-1.68(\mathrm{~m}, 1 \mathrm{H}), 2.39(\mathrm{~s}, 3 \mathrm{H}), 3.31(\mathrm{~d}, 1 \mathrm{H}, J=18.1 \mathrm{~Hz}), 3.36(\mathrm{ddd}, 1 \mathrm{H}, J=8.6,5.3,1.3$ Hz), $3.43(\mathrm{dd}, 1 \mathrm{H}, J=10.8,1.3 \mathrm{~Hz}), 3.49(\mathrm{ap} \mathrm{t}, 1 \mathrm{H}, J=8.6 \mathrm{~Hz}), 3.63(\mathrm{dd}, 1 \mathrm{H}, J=10.0,5.3 \mathrm{~Hz}), 3.71$ $(\mathrm{d}, 1 \mathrm{H}, J=18.3 \mathrm{~Hz}), 3.83(\mathrm{~d}, 1 \mathrm{H}, J=14.4 \mathrm{~Hz}), 5.25(\mathrm{~d}, 1 \mathrm{H}, J=14.4 \mathrm{~Hz}), 7.22-7.31(\mathrm{~m}, 7 \mathrm{H}), 7.54$ (d, $2 \mathrm{H}, J=8.3 \mathrm{~Hz})$. DNOE between $\mathrm{CH}_{3}(1.09) / \mathrm{CH}_{3}(0.36): 5.8 \% ; \mathrm{CH}_{3}(1.09) / \mathrm{CH}(i \mathrm{Pr}): 11.0 \%$; $\mathrm{CH}_{3}$ (1.09) / H-5: 4.6\%; $\mathrm{CH}_{3}(1.09) / \mathrm{H}-3$ (3.71): 3.0\%; $\mathrm{CH}(i \mathrm{Pr}) / \mathrm{H}-3$ (3.71): 3\%; $\mathrm{CH}(i \mathrm{Pr}) / \mathrm{H}-5:$ 1.2\%: CH (iPr) / H-6: 1.2. ${ }^{13}$ C NMR (75 MHz) $\delta-5.3$ (2 C), 18.1, 19.7, 21.0, 21.3, 25.8 (3 C), 26.8, 40.6, 48.7, 57.5, 62.3, 63.6, 125.4 (2 C), 128.1, 128.8 (2 C), 129.1 (2 C), 129.6 (2 C), 136.6, 139.9, 141.8, 165.3. IR (film): $v=3028,2954,2927,2855,1658,1470,1457,1374,1254,1152,1089,955$, 837, 777, $701 \mathrm{~cm}^{-1}$. MS (ES): $537[\mathrm{M}+\mathrm{Na}]^{+}, 515[\mathrm{M}+1]^{+}(100 \%), 375[\mathrm{M}-(p \text { TolSO })]^{+}$. Anal. calcd for $\mathrm{C}_{28} \mathrm{H}_{42} \mathrm{~N}_{2} \mathrm{O}_{3} \mathrm{SSi}$ (514.8): C, 65.33; H, 8.22; N, 5.44; S, 6.23; Si, 5.46; found: C, 65.45; H, 8.07; N, 5.28 .

6.4.

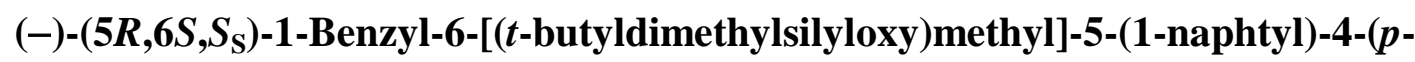
tolylsulfinyl)piperazin-2-one, 8d. 8d (157 mg, $0.263 \mathrm{mmol}, 74 \%)$ was obtained from 7d (226 mg, $0.356 \mathrm{mmol}$ ) as a white solid foam following the general procedure (4 h $30 \mathrm{~min}$ ) and after purification 
by chromatography on silica gel (20-30\% EtO ${ }_{2}$ Ac-hexane). Data for 8d: $\quad \boldsymbol{R}_{\mathbf{f}}=0.15(30 \%$ EtOAchexane). $\mathbf{m p}: 44-46{ }^{\circ} \mathrm{C} . \quad[\alpha]^{20}{ }_{\mathbf{D}}=-122.2(c=0.34) .{ }^{1} \mathbf{H} \mathbf{N M R}(400 \mathrm{MHz}) \delta 0.13(\mathrm{~s}, 3 \mathrm{H}), 0.18$ (s, 3 H), $1.01(\mathrm{~s}, 9 \mathrm{H}), 2.33(\mathrm{~s}, 3 \mathrm{H}), 3.42(\mathrm{~d}, 1 \mathrm{H}, J=17.5 \mathrm{~Hz}), 3.59(\mathrm{dd}, 1 \mathrm{H}, J=9.7,4.8 \mathrm{~Hz}), 3.66$ (dd, 1 $\mathrm{H}, J=9.7,9.5 \mathrm{~Hz}), 3.78(\mathrm{ddd}, 1 \mathrm{H}, J=9.6,4.7,1.9 \mathrm{~Hz}), 3.86(\mathrm{~d}, 1 \mathrm{H}, J=14.4 \mathrm{~Hz}), 4.49(\mathrm{~d}, 1 \mathrm{H}, J=$ $17.5 \mathrm{~Hz}), 4.67(\mathrm{~d}, 1 \mathrm{H}, J=14.4 \mathrm{~Hz}), 5.99(\mathrm{~s}, 1 \mathrm{H}), 6.51(\mathrm{~d}, 2 \mathrm{H}, J=7.1 \mathrm{~Hz}), 6.71(\mathrm{dd}, 2 \mathrm{H}, J=7.7,7.3$ Hz), $6.81(\mathrm{dd}, 1 \mathrm{H}, J=7.5,7.3 \mathrm{~Hz}), 7.20(\mathrm{~d}, 2 \mathrm{H}, J=7.9 \mathrm{~Hz}), 7.29-7.39$ (m, $3 \mathrm{H}), 7.46(\mathrm{~d}, 1 \mathrm{H}, J=7.1$ Hz), $7.52(\mathrm{~d}, 2 \mathrm{H}, J=8.0 \mathrm{~Hz}), 7.69(\mathrm{~d}, 1 \mathrm{H}, J=8.2 \mathrm{~Hz}), 7.75(\mathrm{~d}, 1 \mathrm{H}, J=7.9 \mathrm{~Hz}), 7.82(\mathrm{~d}, 1 \mathrm{H}, J=8.2$ Hz). DNOE between H-5 / Ar-H (7.82): 11\%; H-5 / H-6: 2.1\%; H-5 / 1 H $\left(\mathrm{CH}_{2} \mathrm{O}\right): 2.1 \%$; H-5 / $t \mathrm{Bu}$ : 0.6\%; H-3 / H-3: 25\%; H-3 / Ar-H (7.52): $1 \% ; \mathrm{H}(\mathrm{Bn}) / \mathrm{H}(\mathrm{Bn}): 19 \% ; \mathrm{H}(\mathrm{Bn}) / \mathrm{Ar}-\mathrm{H}(6.51): 1.2 \% .{ }^{13} \mathrm{C}$ NMR (75 MHz) $\delta-5.4,-5.3,18.2,21.3,25.8$ (3 C), 42.7, 49.3, 56.0, 61.4, 62.0, 122.0, 125.3 (2 C), 125.4 (2 C), 125.6, 125.8, 127.2, 127.9 (2 C), 128.0 (2 C), 128.4, 129.0, 129.6 (2 C), 129.7, 133.8, 134.9, 135.1, 139.2, 141.7, 165.3. IR (KBr): $v=3050,2949,2928,2855,1663,1598,1468,1448$, 1255, 1091, 838, $777 \mathrm{~cm}^{-1}$. MS (ES): $1219[2 \mathrm{M}+\mathrm{Na}]^{+}, 1197[2 \mathrm{M}+1]^{+}, 621[\mathrm{M}+\mathrm{Na}]^{+}, 599[\mathrm{M}+1]^{+}$ (100\%), 459 [M-(pTolSO) $]^{+}$. Anal. calcd for $\mathrm{C}_{35} \mathrm{H}_{42} \mathrm{~N}_{2} \mathrm{O}_{3} \mathrm{SSi}$ (598.9): C, 70.19; H, 7.07; N, 4.68; O, 8.01; S, 5.35; Si, 4.69; found: C, 70.22; H, 7.00; N, 4.86.

6.5. (+)-Methyl [(2S,3R, $\left.S_{S}\right)-3-(p$-fluorophenyl)-6-oxo-4-( $p$-tolylsulfinyl)piperazin-2-yl]

carboxylate, 12. 12 (10 mg, $0.026 \mathrm{mmol}, 34 \%)$ was obtained from 11a (33 mg, $0.077 \mathrm{mmol})$ as a colorless oil following the general procedure (40 min) and after purification by chromatography on silica gel $\left(0-5 \% \mathrm{MeOH}-\mathrm{CH}_{2} \mathrm{Cl}_{2}\right)$. Data for 12: $\boldsymbol{R}_{\mathbf{f}}=0.23\left(30 \% \mathrm{MeOH}-\mathrm{Et}_{2} \mathrm{O}\right) . \quad[\alpha]^{\mathbf{2 0}}=+57.1(c=0.70)$. ${ }^{1} \mathbf{H}$ NMR $(300 \mathrm{MHz}) \delta 2.40(\mathrm{~s}, 3 \mathrm{H}), 3.41(\mathrm{~d}, 1 \mathrm{H}, J=17.8 \mathrm{~Hz}), 3.62(\mathrm{~d}, 1 \mathrm{H}, J=17.8 \mathrm{~Hz}), 3.89(\mathrm{~s}, 3$ H), $4.49(\mathrm{dd}, 1 \mathrm{H}, J=4.1,2.2 \mathrm{~Hz}), 5.41(\mathrm{ap} \mathrm{s}, 1 \mathrm{H}), 6.45(\mathrm{~d}, 1 \mathrm{H}, J=3.9 \mathrm{~Hz}), 7.06(\mathrm{t}, 2 \mathrm{H}, J=8.6 \mathrm{~Hz})$, $7.29(\mathrm{~d}, 2 \mathrm{H}, J=8.3 \mathrm{~Hz}), 7.42(\mathrm{~d}, 2 \mathrm{H}, J=8.3 \mathrm{~Hz}), 7.44(\mathrm{~m}, 2 \mathrm{H})$. DNOE between H-3 / 1 Ar-H (7.06): 1.8\%; H-3 / H-2: 5.0\%; H-2 / H-3: 6,2\%; H-2 / 1 Ar-H (7.06): 2.6\%; H-2 / NH: 6.3\%; 2 H-5 / 2Ar-H (7.06): $31 \% .{ }^{13} \mathrm{C}$ NMR $(75 \mathrm{MHz}) \delta 21.4,42.8,53.3,58.5,59.3,116.0\left(\mathrm{~d}, 2 \mathrm{C}, J_{o} \mathrm{C}-\mathrm{F}=22.0\right.$ 
Hz), 125.9 (2 C), $129.3\left(\mathrm{~d}, 2 \mathrm{C}, J_{m} \mathrm{C}-\mathrm{F}=8.2 \mathrm{~Hz}\right), 129.9(2 \mathrm{C}), 131.7,138.9,142.4,162.8\left(\mathrm{~d}, 1 \mathrm{C}, J_{\text {ipso }}\right.$ $\mathrm{C}-\mathrm{F}=241.4 \mathrm{~Hz}), 166.8,170.1 . \quad$ IR (film): $v=3413,2924,1752,1686,1605,1511,1436,1128,1161$, 1091, 1070, 967, $810 \mathrm{~cm}^{-1}$. MS (ES): $803[2 \mathrm{M}+\mathrm{Na}]^{+}, 529[\mathrm{M}+(\mathrm{TolSO})]^{+}, 391[\mathrm{M}+1]^{+}(100 \%), 252$ $[\mathrm{M}-(\mathrm{TolSOH})+1]^{+}$.

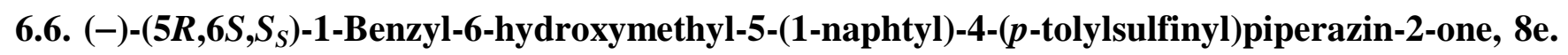
Monoketopiperazine 8e (5 mg, $0.010 \mathrm{mmol}, 31 \%$ ) was obtained as a white oil from chloroacetamide 10e $(17 \mathrm{mg}, 0.033 \mathrm{mmol})$ in $\mathrm{DMF}(0.3 \mathrm{~mL})$ and $\mathrm{Cs}_{2} \mathrm{CO}_{3}(19 \mathrm{mg}, 0.059 \mathrm{mmol})$ according to the general procedure $(4 \mathrm{~h})$ and after purification by chromatography (50-60\% $\left.\mathrm{EtO}_{2} \mathrm{Ac}-\mathrm{hexane}\right)$. Alternatively the title compound has been prepared by other routes: a) To a solution of $\mathbf{6 d}$ (12 $\mathrm{mg}, 0.019 \mathrm{mmol})$ in THF (10 mL/mmol), 1.2 equiv of TBAF $(7 \mathrm{mg}, 0.023 \mathrm{mmol})$ was added and the mixture was stirred from 0 ${ }^{\circ} \mathrm{C}$ to room temperature. After $2 \mathrm{~h}, 0.5$ equiv of TBAF was added and the mixture was stirred until disappearance of starting material ( $4 \mathrm{~h}$ total) monitored by TLC. The reaction was quenched with aqueous saturated $\mathrm{NH}_{4} \mathrm{Cl}$ solution $(4 \mathrm{~mL} / \mathrm{mmol})$ and the aqueous layer was extracted with $\mathrm{CH}_{2} \mathrm{Cl}_{2}(3 \mathrm{x}$ $8 \mathrm{~mL} / \mathrm{mmol})$. The combined organic extracts were washed with a saturated solution of $\mathrm{NaCl}(4$ $\mathrm{mL} / \mathrm{mmol}$ ), dried over $\mathrm{Na}_{2} \mathrm{SO}_{4}$, filtered and concentrated under reduced pressure to give monoketopiperazine $8 \mathbf{e}(8 \mathrm{mg}, 0.016 \mathrm{mmol}, 87 \%)$ after purification by chromatography as a white oil. b) Monoketopiperazine $\mathbf{8 e}(8 \mathrm{mg}, 0.016 \mathrm{mmol}, 97 \%)$ was obtained as a white oil from $8 \mathbf{d}$ (10 mg, 0.017 $\mathrm{mmol})$ in THF $(0.2 \mathrm{~mL})$ and TBAF (6 mg, $0.020 \mathrm{mmol})$, according to the above procedure (a) (45 $\mathrm{min})$ after purification by chromatography. Data for $\mathbf{8 e}: \boldsymbol{R}_{\mathbf{f}}=0.20(60 \%$ EtOAc-hexane $) .[\alpha]^{\mathbf{2 0}}=-58.1(c=$ 0.31). ${ }^{1}$ H NMR (300 MHz) $\delta 2.00(\mathrm{~s}, 3 \mathrm{H}), 3.67(\mathrm{~m}, 3 \mathrm{H}), 3.80(\mathrm{~d}, 1 \mathrm{H}, J=14.4 \mathrm{~Hz}), 4.42(\mathrm{br} \mathrm{s}, 1 \mathrm{H})$, $4.49(\mathrm{~d}, 1 \mathrm{H}, J=16.8 \mathrm{~Hz}), 4.67(\mathrm{~d}, 1 \mathrm{H}, J=14.4 \mathrm{~Hz}), 4.69(\mathrm{~d}, 1 \mathrm{H}, J=16.8 \mathrm{~Hz}), 5.57(\mathrm{~s}, 1 \mathrm{H}), 6.45(\mathrm{~d}$, $2 \mathrm{H}, J=7.1 \mathrm{~Hz}), 6.63(\mathrm{~m}, 2 \mathrm{H}), 6.75(\mathrm{~m}, 1 \mathrm{H}), 6.83(\mathrm{~d}, 2 \mathrm{H}, J=8.0 \mathrm{~Hz}), 7.18-7.42(\mathrm{~m}, 7 \mathrm{H}), 7.57(\mathrm{~d}, 1$ $\mathrm{H}, J=7.6 \mathrm{~Hz}), 7.63(\mathrm{~d}, 1 \mathrm{H}, J=8.1 \mathrm{~Hz}) .{ }^{13} \mathbf{C}$ NMR $(75 \mathrm{MHz}) \delta 21.0,49.4,49.5,50.2,60.3,61.2$, 121.6, 124.7, 124.8, 125.2, 125.7 (2 C), 126.1, 127.2, 127.8 (2 C), 128.0 (2 C), 128.1, 128.7, 129.0, 129.2 (2 C), 133.4, 134.5, 135.0, 137.4, 141.9, 165.0. IR (film): $v=3434,2920,2847,1640,1450$ 
1085, 800, 775, $699 \mathrm{~cm}^{-1} . \quad$ MS (ES): $969[2 \mathrm{M}+1]^{+}, 507[\mathrm{M}+\mathrm{Na}]^{+}(100 \%), 485[\mathrm{M}+1]^{+}, 345$ $[\mathrm{M}-(p \mathrm{TolSO})]^{+}$.

7. General Procedure for the Synthesis of Imino Ketopiperazines 9. A solution of 8 in THF (5 $\mathrm{mL} / \mathrm{mmol})$ was added dropwise to a cold $\left(0^{\circ} \mathrm{C}\right)$ suspension of 4 equiv of $\mathrm{NaH}(60 \%$ in mineral oil $)$ in anhydrous THF $(5 \mathrm{~mL} / \mathrm{mmol}$ of $\mathrm{NaH})$ and the reaction mixture was stirred at room temperature $(1 \mathrm{~h})$ and at reflux $(1 \mathrm{~h})$ until disappearance of starting material was observed by TLC. The mixture was cooled down to room temperature and water $(5 \mathrm{~mL} / \mathrm{mmol}$ of $\mathrm{NaH})$ and $\mathrm{CH}_{2} \mathrm{Cl}_{2}(5 \mathrm{~mL} / \mathrm{mmol})$ were added. The layers were separated and the aqueous phase was extracted with $\mathrm{CH}_{2} \mathrm{Cl}_{2}(3 \times 5 \mathrm{~mL} / \mathrm{mmol})$. The combined organic extracts were washed with a saturated solution of $\mathrm{NaCl}(10 \mathrm{~mL} / \mathrm{mmol})$, dried over $\mathrm{Na}_{2} \mathrm{SO}_{4}$, filtered and concentrated under reduced pressure to give a crude product that was purified by column chromatography on silica gel.

7.1. (+)-(5R,6S)-1-Benzyl-6-(t-butyldimethylsilyloxymethyl)-5-ethyl-5,6-dihydro-1H-pyrazin-2one, 9a. 9a (43 mg, $0.119 \mathrm{mmol}, 83 \%$ ) was obtained from 8a (72 mg, $0.144 \mathrm{mmol})$ as a colorless oil following the general procedure $(1 \mathrm{~h}$ at room temperature and $1 \mathrm{~h}$ at reflux) and after column chromatography on silica gel (30-80\% $\left.\mathrm{Et}_{2} \mathrm{O}-\mathrm{hexane}\right)$. Data for 9a: $\boldsymbol{R}_{\mathbf{f}}=0.38$ (80\% $\left.\mathrm{Et}_{2} \mathrm{O}-\mathrm{hexane}\right)$. $[\alpha]^{20}{ }_{\mathbf{D}}=+188.3(c=1.23) .{ }^{1} \mathbf{H}$ NMR $(500 \mathrm{MHz}) \delta 0.05(\mathrm{~s}, 6 \mathrm{H}), 0.54(\mathrm{t}, 3 \mathrm{H}, J=7.4 \mathrm{~Hz}), 0.86(\mathrm{~s}, 9$ H), 0.86-0.98 (m, $1 \mathrm{H}), 1.40-1.49(\mathrm{~m}, 1 \mathrm{H}), 3.27$ (ap t, $1 \mathrm{H}, J=6.5 \mathrm{~Hz}), 3.55(\mathrm{dd}, 1 \mathrm{H}, J=10.2,6.9$ Hz), $3.63(\mathrm{dd}, 1 \mathrm{H}, J=10.2,5.8 \mathrm{~Hz}), 3.78(\mathrm{dd}, 1 \mathrm{H}, J=7.7,5.5 \mathrm{~Hz}), 3.92(\mathrm{~d}, 1 \mathrm{H}, J=14.3 \mathrm{~Hz}), 5.34$ $(\mathrm{d}, 1 \mathrm{H}, J=14.3 \mathrm{~Hz}), 7.26-7.34(\mathrm{~m}, 5 \mathrm{H}), 7.70(\mathrm{~d}, 1 \mathrm{H}, J=1.5 \mathrm{~Hz}) .{ }^{13} \mathbf{C} \mathbf{N M R}(50 \mathrm{MHz}) \delta-5.6,-5.5$, 10.2, 18.1, 25.8 (4 C,), 48.4, 55.2, 59.4, 63.0, 128.1, 128.8 (2 C), 129.1 (2 C), 136.1, 155.1 (2 C). IR (film): $v=3030,2929,2857,1674,1632,1454,1361,1258,1101,908,838,804,779,704 \mathrm{~cm}^{-1}$. MS (ES): $743[2 \mathrm{M}+\mathrm{Na}]^{+}, 361[\mathrm{M}+1]^{+}(100 \%) .{ }^{1} \mathrm{H}$ and ${ }^{13} \mathrm{C}$ NMR spectral assignments were supported by a 2D NMR technique (HSQC).

7.2. (+)-(5R,6S)-1-Benzyl-6-(t-butyldimethylsilyloxymethyl)-5-(2-phenylethyl)-5,6-dihydro-1Hpyrazin-2-one, 9b. 9b (27 mg, $0.062 \mathrm{mmol}, 87 \%)$ was obtained as a yellow oil from $\mathbf{8 b}(41 \mathrm{mg}, 0.071$ 
mmol) according to the general procedure ( $2 \mathrm{~h} 30 \mathrm{~min})$ and after purification by column chromatography (20-40\% $\mathrm{Et}_{2} \mathrm{O}-$ hexane). Data for $\mathbf{9 b}: \boldsymbol{R}_{\mathbf{f}}=0.28\left(70 \% \mathrm{Et}_{2} \mathrm{O}\right.$-hexane). $[\alpha]^{\mathbf{2 0}}=+243.5$ $(c=2.20) .{ }^{1} \mathbf{H}$ NMR $(300 \mathrm{MHz}) \delta 0.00(\mathrm{~s}, 3 \mathrm{H}), 0.01(\mathrm{~s}, 3 \mathrm{H}), 0.85(\mathrm{~s}, 9 \mathrm{H}), 1.18-1.32(\mathrm{~m}, 1 \mathrm{H}), 1.64-$ $1.76(\mathrm{~m}, 1 \mathrm{H}), 2.28(\mathrm{t}, 2 \mathrm{H}, J=7.8 \mathrm{~Hz}), 3.32($ ap t, $1 \mathrm{H}, J=6.3 \mathrm{~Hz}), 3.52(\mathrm{dd}, 1 \mathrm{H}, J=10.2,7.0 \mathrm{~Hz})$, $3.61(\mathrm{dd}, 1 \mathrm{H}, J=10.3,5.9 \mathrm{~Hz}), 3.91($ ap t, $1 \mathrm{H}, J=7.1 \mathrm{~Hz}), 3.94(\mathrm{~d}, 1 \mathrm{H}, J=14.2 \mathrm{~Hz}), 5.34(\mathrm{~d}, 1 \mathrm{H}, J$ $=14.2 \mathrm{~Hz}), 6.90(\mathrm{~d}, 2 \mathrm{H}, J=8.1 \mathrm{~Hz}), 7.11-7.23(\mathrm{~m}, 3 \mathrm{H}), 7.29-7.36(\mathrm{~m}, 5 \mathrm{H}), 7.74(\mathrm{~d}, 1 \mathrm{H}, J=1.2 \mathrm{~Hz})$.

${ }^{13}$ C NMR (75 MHz) $\delta-5.6$ (2 C), 18.1, 25.8 (3 C), 31.8, 34.3, 48.4, 55.8, 56.9, 62.9, 126.0, 128.1, 128.3 (4 C), 128.9 (2 C), 129.1 (2 C), 136.2, 140.7, 154.9, 155.3. IR (film): v = 3086, 3063, 3028, 2951, 2929, 2857, 1951, 1674, 1627, 1604, 1585, 1496, 1470, 1454, 1390, 1361, 1313, 1257, 1155, 1119, 1030, 1006, 938, 924, 838, 814, 778, 748, 699, $667 \mathrm{~cm}^{-1} . \mathbf{M S}(\mathrm{ES}): 438[\mathrm{M}+2]^{+}, 437[\mathrm{M}+1]^{+}$ (100\%). Anal. calcd for $\mathrm{C}_{26} \mathrm{H}_{36} \mathrm{~N}_{2} \mathrm{O}_{2} \mathrm{Si}$ (436.7): C, 71.51; H, 8.31; N, 6.42; Si, 6.43; found: C, 71.57; H, 8.62; N, 6.53.

\section{3. (+)-(5R,6S)-1-Benzyl-6-(t-butyldimethylsilyloxymethyl)-5-(i-propyl)-5,6-dihydro-1H-pyrazin-}

2-one, 9c. 9c (20 mg, $0.053 \mathrm{mmol}, 84 \%)$ was obtained as a colorless oil from 8c (33 mg, $0.064 \mathrm{mmol})$ according to the general procedure ( $3 \mathrm{~h} 45 \mathrm{~min}$ ) and after purification by column chromatography (20$30 \% \mathrm{Et}_{2} \mathrm{O}$-hexane). Data for 9c: $\boldsymbol{R}_{\mathbf{f}}=0.22\left(60 \% \mathrm{Et}_{2} \mathrm{O}\right.$-hexane $) .[\alpha]^{\mathbf{2 0}}{ }_{\mathbf{D}}=+189.5(c=0.60) .{ }^{\mathbf{1}} \mathbf{H} \mathbf{~ N M R}$ (300 MHz) $\delta 0.00-0.09(\mathrm{~m}, 6 \mathrm{H}), 0.41(\mathrm{~d}, 3 \mathrm{H}, J=6.8 \mathrm{~Hz}), 0.85(\mathrm{~d}, 3 \mathrm{H}, J=6.8 \mathrm{~Hz}), 0.86(\mathrm{~s}, 9 \mathrm{H})$, 1.38-1.48 (m, $1 \mathrm{H}), 3.38(\mathrm{ap} \mathrm{t}, 1 \mathrm{H}, J=6.1 \mathrm{~Hz}), 3.50(\mathrm{dd}, 1 \mathrm{H}, J=10.1,6.7 \mathrm{~Hz}), 3.56(\mathrm{dd}, 1 \mathrm{H}, J=$ 10.3, 5.6 Hz), $3.62($ ap d, $1 \mathrm{H}, J=7.8 \mathrm{~Hz}), 4.01(\mathrm{~d}, 1 \mathrm{H}, J=14.4 \mathrm{~Hz}), 5.21(\mathrm{~d}, 1 \mathrm{H}, J=14.2 \mathrm{~Hz}), 7.30$ $(\mathrm{m}, 5 \mathrm{H}), 7.79(\mathrm{~d}, 1 \mathrm{H}, J=1.5 \mathrm{~Hz}) .{ }^{13} \mathrm{C} \mathbf{N M R}(75 \mathrm{MHz}) \delta-5.6,-5.5,18.1,19.0,19.2,25.8(3 \mathrm{C})$, 32.0, 48.5, 54.4, 63.2, 63.7, 128.1 (2 C), 128.8, 129.3 (2 C), 136.1, 155.0, 155.1. IR (film): $v=3028$, 2949, 2929, 2855, 1676, 1632, 1470, 1257, 1103, 837, $778 \mathrm{~cm}^{-1} . \quad$ MS (ES): $397[\mathrm{M}+\mathrm{Na}]^{+}, 375$ $[\mathrm{M}+1]^{+}(100 \%)$. Anal. calcd for $\mathrm{C}_{21} \mathrm{H}_{34} \mathrm{~N}_{2} \mathrm{O}_{2} \mathrm{Si}$ (374.6): C, 67.33; H, 9.15; N, 7.48; Si, 7.50; found: C, 67.55; H, 9.02; N, 7.23. 2D-NOESY confirmed a cross point H-5/H-6. 
7.4. $\quad(+)-(5 R, 6 S)-1-B e n z y l-6-(t$-butyldimethylsilyloxymethyl)-5-(1-naphtyl)-5,6-dihydro-1H-

pyrazin-2-one, 9d. 9d (9 mg, $0.020 \mathrm{mmol}, 73 \%)$ was obtained as a colorless oil from $8 d$ (16 mg, 0.027 mmol mmol) according to the general procedure $(3 \mathrm{~h})$ and after purification by column chromatography (10-20\% EtOAc-hexane). Data for 9d: $\quad \boldsymbol{R}_{\mathbf{f}}=0.19\left(50 \% \mathrm{Et}_{2} \mathrm{O}-\right.$ hexane $) . \quad[\alpha]^{\mathbf{2 0}}{ }_{\mathbf{D}}=+96.0(c=0.05) .{ }^{\mathbf{1}} \mathbf{H}$ NMR (400 MHz) $\delta 0.11(\mathrm{~s}, 3 \mathrm{H}), 0.12(\mathrm{~s}, 3 \mathrm{H}), 0.97(\mathrm{~s}, 9 \mathrm{H}), 3.72(\mathrm{~m}, 3 \mathrm{H}), 3.90(\mathrm{~d}, 1 \mathrm{H}, J=14.5 \mathrm{~Hz})$, $4.82(\mathrm{~d}, 1 \mathrm{H}, J=14.5 \mathrm{~Hz}), 6.29($ ap s, $1 \mathrm{H}), 6.60(\mathrm{~d}, 2 \mathrm{H}, J=7.3 \mathrm{~Hz}), 6.77$ (t, $2 \mathrm{H}, J=7.6 \mathrm{~Hz}), 6.88$ (m, $2 \mathrm{H}), 7.30($ ap t, $1 \mathrm{H}), 7.43(\mathrm{~m}, 2 \mathrm{H}), 7.73(\mathrm{~d}, 1 \mathrm{H}, J=8.2 \mathrm{~Hz}), 7.82(\mathrm{dd}, 1 \mathrm{H}, J=7.3,2.5 \mathrm{~Hz}), 8.07$ (d, $1 \mathrm{H}, J=7.5 \mathrm{~Hz}), 8.26(\mathrm{~d}, 1 \mathrm{H}, J=1.6 \mathrm{~Hz})$. DNOE between Ar-H (8.07) / Ar-H (7.43): 7\%; Ar-H (8.07) / H-5: 7\%; Ar-H (8.07) / H-6: 2\%; Ar-H (8.07) / $\mathrm{CH}_{2} \mathrm{O}: 4 \%$; H-5 / Ar-H (8.07): 16\%; H-5 / $\mathrm{CH}_{2} \mathrm{O}: 4 \%$; H-5 / H-6: 2\%. ${ }^{13} \mathrm{C}$ NMR (100 MHz) $\delta-5.5$ (2 C), 18.3, 25.8 (3 C), 48.6, 57.2, 58.7, 62.5, $122.5,124.4,124.9,125.5,126.2,127.3,127.9$ (2 C), 128.0 (2 C), 128.5, 129.1, 130.0, 131.4 134.3, 134.8, 155.3, 158.4. IR (film): $v=3026,2951,2925,2855,1678,1461,1255,1100,1028,836,778$ $\mathrm{cm}^{-1}$. MS (ES): $939[2 \mathrm{M}+\mathrm{Na}]^{+}, 459[\mathrm{M}+1]^{+}(100 \%)$. Anal. calcd for $\mathrm{C}_{28} \mathrm{H}_{34} \mathrm{~N}_{2} \mathrm{O}_{2} \mathrm{Si}(458.7)$ : C, 73.32 ; H, 7.47; N, 6.11; Si, 6.12; found: C, 73.52; H, 7.10; N, 6.31 .

\section{Nucleophilic Addition Reactions to Imino Monoketopiperazines.}

The tentative stereochemical assignment for $\mathbf{1 3 a}-\mathbf{c}$ and $\mathbf{1 4 a - c}$ is based on the comparison $\left({ }^{1} \mathrm{H} \mathrm{NMR}\right)$ with of 13d and 14d assigned by DNOE. In general for compounds $\mathbf{1 3}$, the chemical shift of one of the benzylic protons is higher than for compounds 14 (4.00-3.91 ppm vs 3.92-3.82 ppm) and the chemical

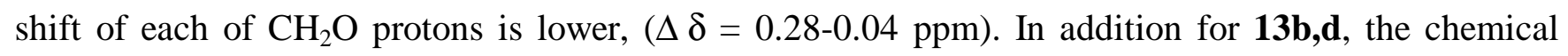
shifts of $\underline{\mathrm{CH}} i \mathrm{Pr}$ are lower than for $\mathbf{1 4 b , c}(\Delta \delta=0.41-0.17 \mathrm{ppm})$.

8.1. General Procedure for the Addition of Potassium Cyanide. ${ }^{3}$ To a solution of 1 equiv of imino piperazinone 9 in THF $(1.5 \mathrm{~mL} / \mathrm{mmol})$ was added 3.0 equiv of HOAc and 3.0 equiv of a $4.8 \mathrm{M}$ aqueous solution of KCN. The mixture was stirred at room temperature for $20 \mathrm{~h}$ (monitored by TLC), and then 1 equiv of HOAc and 1 equiv of $\mathrm{KCN}(13 \mu \mathrm{L}, 0.064 \mathrm{mmol})$ was added. After a total of $26-28 \mathrm{~h}$ the crude mixture was basified to $\mathrm{pH}=10-11$ with solid $\mathrm{K}_{2} \mathrm{CO}_{3}$, concentrated under reduced pressure and re- 
disolved in $\mathrm{CH}_{2} \mathrm{Cl}_{2}(10 \mathrm{~mL} / \mathrm{mmol})$ and $\mathrm{H}_{2} \mathrm{O}(10 \mathrm{~mL} / \mathrm{mmol})$. The aqueous phase was extracted with $\mathrm{CH}_{2} \mathrm{Cl}_{2}(2 \times 10 \mathrm{~mL} / \mathrm{mmol})$ and the combined organic layers were washed with a saturated solution of $\mathrm{NaHCO}_{3}$, dried over $\mathrm{Na}_{2} \mathrm{SO}_{4}$ and concentrated under reduced pressure. The crude was purified by column chromatography using the appropriate mixture of eluents.

\subsection{1. (5S,6R)-4-Benzyl-5-[(t-butyldimethylsilyloxy)methyl]-3-oxo-6-(2-phenylethyl) piperazin-2-}

yl]carbonitrile, 13a and 14a. From $9 b(28 \mathrm{mg}, 0.064 \mathrm{mmol})$, HOAc, and $\mathrm{KCN}$ according to the general procedure was obtained a crude mixture that was purified by column chromatography $(20-50 \%$ $\mathrm{Et}_{2} \mathrm{O}$-hexane). Amino nitriles 13a and 14a (12 mg, $\left.0.026 \mathrm{mmol}, 40 \%\right)$ were isolated as a 69:31 mixture along with recovered starting material $(6 \mathrm{mg}, 0.014 \mathrm{mmol}, 21 \%)$. The stereochemical assignment is tentative. Data of major isomer 13a from the mixture: $\boldsymbol{R}_{\mathbf{f}}=0.24$ (70\% $\mathrm{Et}_{2} \mathrm{O}$-hexane). ${ }^{\mathbf{1}} \mathbf{H}$ NMR $(300$ MHz) $\delta 0.01(\mathrm{~s}, 3 \mathrm{H}), 0.02(\mathrm{~s}, 3 \mathrm{H}), 0.90(\mathrm{~s}, 9 \mathrm{H}), 1.47-1.73(\mathrm{~m}, 2 \mathrm{H}), 2.41-2.64(\mathrm{~m}, 2 \mathrm{H}), 3.00-3.24$ (m, $2 \mathrm{H}), 3.66(\mathrm{dd}, 1 \mathrm{H}, J=10.6,3.1 \mathrm{~Hz}), 3.88(\mathrm{dd}, 1 \mathrm{H}, J=10.7,5.3 \mathrm{~Hz}), 3.97(\mathrm{~d}, 1 \mathrm{H}, J=14.4 \mathrm{~Hz})$, $4.52(\mathrm{~s}, 1 \mathrm{H}), 5.43(\mathrm{~d}, 1 \mathrm{H}, J=14.4 \mathrm{~Hz}), 7.02-7.06(\mathrm{~m}, 2 \mathrm{H}), 7.16-7.38(\mathrm{~m}, 8 \mathrm{H}) .{ }^{\mathbf{1 3}} \mathbf{C} \mathbf{N M R}(75 \mathrm{MHz})$ $\delta-5.5$ (2 C), 18.2, 25.9 (3 C), 32.0, 32.9, 47.8, 48.7, 51.3, 59.1, 62.8, 118.1, 126.1, 128.2-128.9 (9 C), 135.9, 140.7, 161.7. IR (film): $v=3333,2928,2847,2413,1736,1662,1454,1255,1116,959,829$, 775, $702 \mathrm{~cm}^{-1}$. MS (ES): $949[2 \mathrm{M}+\mathrm{Na}]^{+}, 464[\mathrm{M}+1]^{+}(100 \%)$. Partial data of minor isomer 14a from the mixture: $\boldsymbol{R}_{\mathbf{f}}=0.24\left(70 \% \mathrm{Et}_{2} \mathrm{O}-\right.$ hexane $) .{ }^{1} \mathbf{H}$ NMR $(300 \mathrm{MHz}) \delta 3.16-3.22(\mathrm{~m}, 2 \mathrm{H}), 3.62(\mathrm{dd}, 1 \mathrm{H}$, $J=10.5,3.2 \mathrm{~Hz}), 3.79(\mathrm{dd}, 1 \mathrm{H}, J=10.7,5.6 \mathrm{~Hz}), 3.92(\mathrm{~d}, 1 \mathrm{H}, J=14.4 \mathrm{~Hz}), 5.43(\mathrm{~d}, 1 \mathrm{H}, J=14.4$ Hz). ${ }^{13}$ C NMR (75 MHz) $\delta-5.6$ (2 C), 48.5, 51.0, 59.4, 62.2, 116.4, 161.8.

\subsection{2. $\quad(5 S, 6 R)-4-B e n z y l-5-[(t$-butyldimethylsilyloxy $)$ methyl]-3-oxo-6-( $i$-propyl)piperazin-2-}

yl]carbonitrile, 13b and 14b. From 9c (19 mg, $0.051 \mathrm{mmol})$, HOAc, and $\mathrm{KCN}$ according to the general procedure was obtained a crude mixture that was purified by column chromatography (10-30\% $\mathrm{Et}_{2} \mathrm{O}$-hexane). Amino nitriles 13b and 14b (13 $\left.\mathrm{mg}, 0.032 \mathrm{mmol}, 63 \%\right)$ were isolated as a 64:36 mixture along with recovered starting material $(2 \mathrm{mg}, 0.005 \mathrm{mmol}, 10 \%)$. Data of mayor isomer $\mathbf{1 3 b}$ from the mixture: $\boldsymbol{R}_{\mathbf{f}}=0.25\left(50 \% \mathrm{Et}_{2} \mathrm{O}-\right.$ hexane)$){ }^{1} \mathbf{H}$ NMR $(400 \mathrm{MHz}) \delta 0.03(\mathrm{~s}, 3 \mathrm{H}), 0.07(\mathrm{~s}, 3 \mathrm{H}), 0.49(\mathrm{~d}, 3$ 
$\mathrm{H}, J=6.6 \mathrm{~Hz}), 0.84(\mathrm{~d}, 3 \mathrm{H}, J=6.6 \mathrm{~Hz}), 0.89(\mathrm{~s}, 9 \mathrm{H}), 1.52-1.60(\mathrm{~m}, 1 \mathrm{H}), 2.36(\mathrm{br} \mathrm{s}, 1 \mathrm{H}), 2.69(\mathrm{dd}, 1$ $\mathrm{H}, J=8.7,3.0 \mathrm{~Hz}), 3.24(\mathrm{dt}, 1 \mathrm{H}, J=5.5,3.5 \mathrm{~Hz}), 3.73(\mathrm{dd}, 1 \mathrm{H}, J=10.5,3.7 \mathrm{~Hz}), 3.89(\mathrm{dd}, 1 \mathrm{H}, J=$ 10.4, $5.7 \mathrm{~Hz}), 3.91(\mathrm{~d}, 1 \mathrm{H}, J=14.7 \mathrm{~Hz}), 4.49(\mathrm{~s}, 1 \mathrm{H}), 5.45(\mathrm{~d}, 1 \mathrm{H}, J=14.7 \mathrm{~Hz}), 7.23-7.35(\mathrm{~m}, 5 \mathrm{H})$. ${ }^{13}$ C NMR (75 MHz) $\delta$-5.5 (2 C), 18.2 (2 C), 19.5, 25.8 (3 C), 26.7, 48.3, 48.5, 56.8, 57.5, 63.3, 116.6, 128.0-128.8 (3 C), 128.9 (2 C), 136.1, 161.7. Data of minor isomer $\mathbf{1 4 b}$ from the mixture: $\boldsymbol{R}_{\mathbf{f}}=0.25$ (50\% $\mathrm{Et}_{2} \mathrm{O}$-hexane). ${ }^{1} \mathrm{H}$ NMR $(400 \mathrm{MHz}) \delta 0.04(\mathrm{~s}, 3 \mathrm{H}), 0.07(\mathrm{~s}, 3 \mathrm{H}), 0.36(\mathrm{~d}, 3 \mathrm{H}, J=6.6 \mathrm{~Hz}), 0.87$ (s, $9 \mathrm{H}), 0.98(\mathrm{~d}, 3 \mathrm{H}, J=6.6 \mathrm{~Hz}), 1.73-1.78(\mathrm{~m}, 1 \mathrm{H}), 2.36(\mathrm{br} \mathrm{s}, 1 \mathrm{H}), 2.57(\mathrm{dd}, 1 \mathrm{H}, J=9.5,2.2 \mathrm{~Hz})$, $3.29(\mathrm{dt}, 1 \mathrm{H}, J=5.5,2.7 \mathrm{~Hz}), 3.35(\mathrm{dd}, 1 \mathrm{H}, J=10.4,3.3 \mathrm{~Hz}), 3.82(\mathrm{dd}, 1 \mathrm{H}, J=10.6,5.5 \mathrm{~Hz}), 3.82$ $(\mathrm{d}, 1 \mathrm{H}, J=14.3 \mathrm{~Hz}), 4.49(\mathrm{~s}, 1 \mathrm{H}), 5.46(\mathrm{~d}, 1 \mathrm{H}, J=14.3, \mathrm{~Hz}), 7.23-7.35(\mathrm{~m}, 5 \mathrm{H}) .{ }^{13} \mathbf{C} \mathbf{N M R}(75$ MHz) $\delta$-5.5 (2 C), 18.2, 19.2, 19.5, 25.8 (3 C), 27.6, 48.3, 48.5, 56.4, 58.8, 63.3, 117.6, 128.0-128.8 (3 C), 128.9 (2 C), 136.1, 162.4. IR (film): $v=3333,2929,2862,2406,1662,1451,1465,1299$, 1248, 1105, 956, 837, 782, $699 \mathrm{~cm}^{-1}$. MS (ES): $825[2 \mathrm{M}+\mathrm{Na}]^{+}(100 \%), 424[\mathrm{M}+\mathrm{Na}]^{+}, 402[\mathrm{M}+1]^{+}$, $375[\mathrm{M}-\mathrm{CN}]^{+}$.

\subsection{Addition of Diethylzinc. ${ }^{4}(3 S, 5 R, 6 S)$-1-Benzyl-6-[(t-butyldimethylsilyloxy)methyl]-3-ethyl-5-} (2-phenylethyl)piperazin-2-one, $13 \mathrm{c}$ and $(3 R, 5 R, 6 S)$-1-Benzyl-6-[( $t$-butyldimethylsilyloxy)methyl]3-ethyl-5-(2-phenylethyl)piperazin-2-one, 14c. A solution of $9 \mathbf{b}(29 \mathrm{mg}, 0.066 \mathrm{mmol})$ in toluene $(6.25 \mathrm{~mL} / \mathrm{mmol}), 1.25$ equiv of TMSCl $(9 \mathrm{mg}, 11 \mu \mathrm{L}, 0.083 \mathrm{mmol})$ and 2.5 equiv of $\mathrm{Et}_{2} \mathrm{Zn}(1 \mathrm{M}$ in hexane; $0.17 \mathrm{~mL}, 0.165 \mathrm{mmol}$ ) was stirred from $0{ }^{\circ} \mathrm{C}$ to room temperature during $21 \mathrm{~h}$. Then, 0.5 equiv of TMSCl (4 mg, $4 \mu \mathrm{L}, 0.033 \mathrm{mmol})$ and 1 equiv of $\mathrm{Et}_{2} \mathrm{Zn}(0.07 \mathrm{~mL}, 0.066 \mathrm{mmol})$ was added. The evolution of the mixture was monitored by TLC and after 2 days was quenched with $\mathrm{NH}_{4} \mathrm{Cl}(6.26$ $\mathrm{mL} / \mathrm{mmol})$. The aqueous layer was extracted with $\mathrm{CH}_{2} \mathrm{Cl}_{2}(3 \times 10 \mathrm{~mL} / \mathrm{mmol})$ and the combined organic extracts were dried over $\mathrm{MgSO}_{4}$, filtered and concentrated under reduced pressure to obtain after column chromatography (5-30\% $\mathrm{Et}_{2} \mathrm{O}$-hexane) an inseparable mixture (78:22) of 3-ethylpiperazin-2ones $13 \mathrm{c}$ and $14 \mathrm{c}(13 \mathrm{mg}, 0.028 \mathrm{mmol}, 42 \%)$ as a colorless oil and starting material (15 mg, 0.034 mmol, 52\%). Data of $\mathbf{1 3 c}$ from the mixture: $\boldsymbol{R}_{\mathbf{f}}=0.22\left(60 \% \mathrm{Et}_{2} \mathrm{O}-\right.$ hexane). ${ }^{1} \mathbf{H}$ NMR $(300 \mathrm{MHz})$ 
$\delta-0.05-0.00(\mathrm{~m}, 6 \mathrm{H}), 0.84-0.86(\mathrm{~m}, 9 \mathrm{H}), 1.00(\mathrm{t}, 3 \mathrm{H}, J=7.3 \mathrm{~Hz}), 1.58-1.74(\mathrm{~m}, 4 \mathrm{H}), 2.10(\mathrm{qd}, 1 \mathrm{H}$, $J=7.6,4.1 \mathrm{~Hz}), 2.56-2.68(\mathrm{~m}, 2 \mathrm{H}), 3.03(\mathrm{~m}, 2 \mathrm{H}), 3.17(\mathrm{dd}, 1 \mathrm{H}, J=8.1,4.2 \mathrm{~Hz}), 3.47(\mathrm{dd}, 1 \mathrm{H}, J=$ 10.8, $2.8 \mathrm{~Hz}), 3.68(\mathrm{dd}, 1 \mathrm{H}, J=10.8,3.8 \mathrm{~Hz}), 3.96(\mathrm{~d}, 1 \mathrm{H}, J=15.1 \mathrm{~Hz}), 5.38(\mathrm{~d}, 1 \mathrm{H}, J=15.1 \mathrm{~Hz})$, 7.06-7.32 (m, $10 \mathrm{H}) .{ }^{1} \mathbf{H}$ NMR $\left(\mathrm{CD}_{3} \mathrm{OD}, 300 \mathrm{MHz}\right) \delta-0.05(\mathrm{~s}, 3 \mathrm{H}), 0.08(\mathrm{~s}, 3 \mathrm{H}), 0.83(\mathrm{~s}, 9 \mathrm{H}), 1.00$ (t, $3 \mathrm{H}, J=7.4 \mathrm{~Hz}), 1.64-1.73(\mathrm{~m}, 3 \mathrm{H}), 1.99(\mathrm{qd}, 1 \mathrm{H}, J=7.6,4.4 \mathrm{~Hz}), 2.63(\mathrm{td}, 2 \mathrm{H}, J=7.3,2.2 \mathrm{~Hz})$, $3.01(\mathrm{~m}, 1 \mathrm{H}), 3.13-3.19(\mathrm{~m}, 2 \mathrm{H}), 3.49(\mathrm{dd}, 1 \mathrm{H}, J=11.2,2.4 \mathrm{~Hz}), 3.76(\mathrm{dd}, 1 \mathrm{H}, J=11.2,3.7 \mathrm{~Hz})$ $4.12(\mathrm{~d}, 1 \mathrm{H}, J=15.1 \mathrm{~Hz}), 5.13(\mathrm{~d}, 1 \mathrm{H}, J=15.1 \mathrm{~Hz}), 6.79(\mathrm{~d}, 1 \mathrm{H}, J=6.8 \mathrm{~Hz}), 7.09-7.35(\mathrm{~m}, 9 \mathrm{H})$. ${ }^{13}$ C NMR (75 MHz) $\delta$-5.6 (2 C), 10.7, 18.1, 25.4, 25.8 (3 C), 32.5, 36.3, 47.0, 53.1, 59.0, 61.4, 61.6, 126.0, 127.3, 128.1 (2 C), 128.3 (2 C), 128.5 (2 C), 128.6 (2 C), 137.5, 141.5, 173.1. IR (film): v = $3329,3063,3027,2953,2928,2856,1645,1604,1495,1453,1360,1314,1257,1111,1029,1006$, 972, 938, 837, 812, 777, 748, 699, $669 \mathrm{~cm}^{-1}$. MS (ES): $467[\mathrm{M}+1]^{+}(100 \%)$. Partial data of 14c from the mixture: $\boldsymbol{R}_{\mathbf{f}}=0.22\left(60 \% \mathrm{Et}_{2} \mathrm{O}-\right.$ hexane $) .{ }^{1} \mathbf{H}$ NMR $(300 \mathrm{MHz}) \delta 3.81(\mathrm{dd}, 1 \mathrm{H}, J=10.2,7.2 \mathrm{~Hz})$, $3.90(\mathrm{~d}, 1 \mathrm{H}, J=14.6 \mathrm{~Hz}), 5.36(\mathrm{~d}, 1 \mathrm{H}, J=14.4 \mathrm{~Hz}) .{ }^{1} \mathbf{H} \mathbf{N M R}\left(\mathrm{CD}_{3} \mathrm{OD}, 300 \mathrm{MHz}\right) \delta 1.10(\mathrm{t}, 3 \mathrm{H}, J=$ $7.4 \mathrm{~Hz}), 3.42(\mathrm{dd}, 1 \mathrm{H}, J=7.1,4.0 \mathrm{~Hz}), 3.62(\mathrm{dd}, 1 \mathrm{H}, J=11.5,6.6 \mathrm{~Hz}), 3.89(\mathrm{dd}, 1 \mathrm{H}, J=10.3,6.6$ Hz), $4.08(\mathrm{~d}, 1 \mathrm{H}, J=14.6 \mathrm{~Hz}), 5.22(\mathrm{~d}, 1 \mathrm{H}, J=14.4 \mathrm{~Hz}) .{ }^{13} \mathbf{C}$ NMR $(75 \mathrm{MHz}) \delta 10.2,54.9,59.7$, $63.4,170.9$

\subsection{Allylations.}

8.3.1. Procedure of Addition of allyltributylstannane and $\mathbf{T i C l}_{\mathbf{4}}$. A solution of $\mathbf{9 c}(28 \mathrm{mg}, 0.075$ mmol $)$ in $\mathrm{CH}_{2} \mathrm{Cl}_{2}(5 \mathrm{~mL} / \mathrm{mmol})$ and 1 equiv of $\mathrm{TiCl}_{4}(14 \mathrm{mg}, 8 \mu \mathrm{L}, 0.075 \mathrm{mmol})$ was stirred at $-78^{\circ} \mathrm{C}$ for $3 \mathrm{~h}$ and then 2 equiv of allyltributylstannane $(50 \mathrm{mg}, 46 \mu \mathrm{L}, 0.150 \mathrm{mmol})$ was added. After $1 \mathrm{~h}$, the mixture was warmed up to room temperature and monitored by TLC (48 h). Then the mixture was hydrolyzed with $2 \mathrm{~N}$ aqueous $\mathrm{NaOH}(10 \mathrm{~mL} / \mathrm{mmol})$. The solvent was evaporated in vacuo and the residue was extracted with $\mathrm{CH}_{2} \mathrm{Cl}_{2}(4 \times 10 \mathrm{~mL} / \mathrm{mmol})$. The organic extracts were washed with a saturated solution of $\mathrm{NaHCO}_{3}$, dried over $\mathrm{Na}_{2} \mathrm{SO}_{4}$, filtered and concentrated in vacuo to afford $\mathbf{1 3 d}$ and 
14d as a 43:57 mixture. The combined yield of the mixture after purification by column chromatography (5-10\% EtOAc-hexane) was 77\% (24 mg, $0.058 \mathrm{mmol})$. Small amounts of 13d and 14d were separated after careful chromatography on silica gel.

8.3.2. Procedure of Addition of allyltrimethylsilane and $\mathbf{S n C l}_{\mathbf{4}} \cdot{ }^{\mathbf{5}}$ Allyltrimethylsilane $(12 \mathrm{mg}, 17 \mu \mathrm{L}$, $0.106 \mathrm{mmol})$ and $\mathrm{SnCl}_{4}(51 \mathrm{mg}, 23 \mu \mathrm{L}, 0.194 \mathrm{mmol})$ were added to a cold $\left(0^{\circ} \mathrm{C}\right)$ solution of $9 \mathrm{c}(33 \mathrm{mg}$, $0.088 \mathrm{mmol})$ in THF $(10 \mathrm{~mL} / \mathrm{mmol})$. The mixture was stirred for $1 \mathrm{~h}$ at $0{ }^{\circ} \mathrm{C}$ and warmed up to room temperature. After 5 days (monitored by TLC) the mixture was hydrolyzed with $2 \mathrm{~N}$ aqueous $\mathrm{NaOH}(10$ $\mathrm{mL} / \mathrm{mmol})$. The solvent was evaporated in vacuo and the residue was extracted 4 times with $\mathrm{CH}_{2} \mathrm{Cl}_{2}(10$ $\mathrm{mL} / \mathrm{mmol}$ ). The organic extracts were washed with a saturated solution of $\mathrm{NaHCO}_{3}$, dried over $\mathrm{Na}_{2} \mathrm{SO}_{4}$, filtered and concentrated in vacuum to yield after purification by column chromatography (5-10\% EtOAc-hexane) a mixture of $\mathbf{1 3 d}$ and $\mathbf{1 4 d}(76: 24)(27 \mathrm{mg}, 0.065 \mathrm{mmol}, 74 \%)$ as a colorless oil.

8.3.3. Procedure of Addition of Allylmagnesium Bromide and $\mathbf{B F}_{\mathbf{3}} \cdot \mathbf{O E t}_{2} \cdot 2$ Equiv of $\mathrm{BF}_{3} \cdot \mathrm{OEt}_{2}$ was added to a cold $\left(-78{ }^{\circ} \mathrm{C}\right)$ solution of $9 \mathrm{c}(31 \mathrm{mg}, 0.082 \mathrm{mmol})$ in $\mathrm{THF}(10 \mathrm{~mL} / \mathrm{mmol})$. The mixture was stirred for $1 \mathrm{~h}$ and then 2 equiv of allylmagnesium bromide $0.64 \mathrm{M}$ solution $(0.26 \mathrm{~mL}, 0.166 \mathrm{mmol})$ in $\mathrm{Et}_{2} \mathrm{O}$ was added. The reaction mixture was stirred at low temperature $21 \mathrm{~h}$ (TLC) and then was quenched with $\mathrm{NH}_{4} \mathrm{Cl}(5 \mathrm{~mL} / \mathrm{mmol})$ and $\mathrm{H}_{2} \mathrm{O}(5 \mathrm{~mL} / \mathrm{mmol})$. The layers were separated and the aqueous phase was extracted with $\mathrm{CH}_{2} \mathrm{Cl}_{2}(3 \times 5 \mathrm{~mL} / \mathrm{mmol})$. The combined organic extracts were washed with a saturated solution of $\mathrm{NaCl}$, dried over $\mathrm{Na}_{2} \mathrm{SO}_{4}$, filtered and concentrated under reduced pressure to give a mixture (24:76) of $\mathbf{1 3 d}$ and $\mathbf{1 4 d}(22 \mathrm{mg}, 0.053 \mathrm{mmol}, 64 \%)$ after column chromatography (5-10\% EtOAc-hexane) as a colorless oil and recovered starting material (18\%).

8.3.4. Procedure of Addition of Allylmagnesium Bromide and $\mathbf{C e C l}_{3}$. Anhydrous $\mathrm{CeCl}_{3}(38 \mathrm{mg}$, $0.154 \mathrm{mmol})^{6}$ was stirred in THF $(0.77 \mathrm{~mL}, 5 \mathrm{~mL} / \mathrm{mmol}) 45 \mathrm{~min}$ at $\mathrm{rt}$. Then at $-45^{\circ} \mathrm{C}, 2.5$ equiv of 0.38 $\mathrm{mL}$ of a $0.40 \mathrm{M}$ solution of allylmagnesium bromide $(0.154 \mathrm{mmol})$ in $\mathrm{Et}_{2} \mathrm{O}$ was added. The mixture was cooled to $-78{ }^{\circ} \mathrm{C}$ and a solution of $9 \mathrm{c}(23 \mathrm{mg}, 0.061 \mathrm{mmol})$ in $\mathrm{THF}(2 \mathrm{~mL} / \mathrm{mmol})$ was slowly added. The mixture was stirred to room temperature for $7 \mathrm{~h}$ and then 1.5 equiv of $\mathrm{CeCl}_{3}(23 \mathrm{mg}, 0.091$ 
mmol) and allylmagnesium bromide $(0.23 \mathrm{~mL}, 0.091 \mathrm{mmol})$ was added to the mixture. The evolution was followed by TLC (total $22 \mathrm{~h} 30 \mathrm{~min})$ and the reaction was quenched with $\mathrm{NH}_{4} \mathrm{Cl}(5 \mathrm{~mL} / \mathrm{mmol})$. The layers were separated and the aqueous phase was extracted with $\mathrm{CH}_{2} \mathrm{Cl}_{2}(3 \times 5 \mathrm{~mL} / \mathrm{mmol})$. The combined organic extracts were washed with a saturated solution of $\mathrm{NaCl}$, dried over $\mathrm{Na}_{2} \mathrm{SO}_{4}$, filtered and concentrated under reduced pressure to give just isomer 14d (7 mg, $0.017 \mathrm{mmol}, 28 \%)$ after column chromatography (5-10\% EtOAc-hexane) as a colorless oil and starting material (12 mg, 0.032 mmol, 53\%).

8.3.5. Procedure for Zinc-Mediated Barbier Allylation. ${ }^{6}$ To a suspension of Zn powder (2 equiv), (12 mg, $0.176 \mathrm{mmol})$ in THF $(1.5 \mathrm{ml} / \mathrm{mmol})$ at $0{ }^{\circ} \mathrm{C}$, was added $\mathrm{CeCl}_{3} \cdot 7 \mathrm{H}_{2} \mathrm{O}(0.1$ equiv, $3 \mathrm{mg}, 0.009$ mmol) and a solution of 9c (1 equiv) and 1.5 equiv of allyl bromide in THF $(3.0 \mathrm{~mL} / \mathrm{mmol} 9 \mathbf{c})$. The mixture was stirred from $0{ }^{\circ} \mathrm{C}$ to room temperature monitored by TLC (23-24 h) and then was quenched with $\mathrm{NH}_{4} \mathrm{Cl}$ (6 mL/mmol). The layers were separated and the aqueous phase was extracted with $\mathrm{CH}_{2} \mathrm{Cl}_{2}$ $(3 \times 10 \mathrm{~mL} / \mathrm{mmol})$. The combined organic extracts were washed with a saturated solution of $\mathrm{NaCl}$, dried over $\mathrm{Na}_{2} \mathrm{SO}_{4}$, filtered and concentrated under reduced pressure to give the final products (14) after purification by column chromatography. The same procedure was applied in the absence of the $\mathrm{CeCl}_{3} \cdot 7 \mathrm{H}_{2} \mathrm{O}$ with complete recovery of the starting material.

(+)-(3R,5R,6S)-3-Allyl-1-benzyl-6-[( $t$-butyldimethylsilyloxy)methyl]-5-(i-propyl)piperazin-2-one,

14d. From 9c (33 mg, $0.088 \mathrm{mmol})$ and 1.5 equiv of allyl bromide (11 $\mu \mathrm{L}, 0.132 \mathrm{mmol})$ was obtained after chromatography (5-10\% EtOAc-hexane) 14d (32 mg, $0.077 \mathrm{mmol}, 87 \%)$ as a colorless oil. Data for 14d: $\boldsymbol{R}_{\mathbf{f}}=0.34\left(20 \%\right.$ EtOAc-hexane). $[\alpha]^{\mathbf{2 0}}{ }_{\mathbf{D}}=+77.0(c=1.33) .{ }^{\mathbf{1}} \mathbf{H} \mathbf{N M R}(400 \mathrm{MHz}) \delta 0.04(\mathrm{~s}, 6$ H), $0.34(\mathrm{~d}, 3 \mathrm{H}, J=6.6 \mathrm{~Hz}), 0.85(\mathrm{~d}, 3 \mathrm{H}, J=6.6 \mathrm{~Hz}), 0.87(\mathrm{~s}, 9 \mathrm{H}), 1.58(\mathrm{~m}, 1 \mathrm{H}), 1.73(\mathrm{br}, 1 \mathrm{H})$, $2.40($ ap d, $1 \mathrm{H}, J=9.3 \mathrm{~Hz}), 2.45($ ap t, $1 \mathrm{H}, J=7.7 \mathrm{~Hz}), 2.68(\mathrm{~m}, 1 \mathrm{H}), 3.24(\mathrm{ddd}, 1 \mathrm{H}, J=7.7,4.8,1.1$ Hz), $3.49(\mathrm{dd}, 1 \mathrm{H}, J=8.1,3.3 \mathrm{~Hz}), 3.73(\mathrm{dd}, 1 \mathrm{H}, J=9.9,4.8 \mathrm{~Hz}), 3.81(\mathrm{dd}, 1 \mathrm{H}, J=9.9,7.7 \mathrm{~Hz})$, $3.82(\mathrm{~d}, 1 \mathrm{H}, J=14.3 \mathrm{~Hz}), 5.11(\mathrm{dd}, 1 \mathrm{H}, J=10.1,1.1 \mathrm{~Hz}), 5.16(\mathrm{dd}, 1 \mathrm{H}, J=17.0,1.4 \mathrm{~Hz}), 5.44(\mathrm{~d}, 1$ $\mathrm{H}, J=14.3 \mathrm{~Hz}$ ), 5.77 (ddt, $1 \mathrm{H}, J=17.0,10.1,7.3 \mathrm{~Hz}$ ), 7.25-7.29 (m, $5 \mathrm{H})$. DNOE between H-3 / H-2 
allyl: 1.4\%; H-3 / $1 \mathrm{H} \mathrm{CH}_{2}$ allyl: 3.5\%; H-3 / $1 \mathrm{H} \mathrm{CH}_{2}$ allyl: $2.0 \%$; $\mathrm{H}-3$ / $\mathrm{CH} i \operatorname{Pr}: 5.9 \%$; $\mathrm{CH} i \operatorname{Pr} / \mathrm{H}-3$ : 1.8\%; CH $i \mathrm{Pr} / \mathrm{H}-6: 2.4 \%$; H-6 / 1 H CH $\mathrm{CH}_{2} \mathrm{Ph}: 1 \%$; H-6 / 1 H CH $\mathrm{CH}_{2} \mathrm{Ph}: 5 \%$; H-6 / $1 \mathrm{H} \mathrm{CH}_{2} \mathrm{O}: 4 \% .{ }^{13} \mathrm{C}$ NMR (75 MHz) $\delta-5.4,-5.3,18.2,19.2,19.5,25.4,25.9$ (3 C), 37.7, 48.7, 53.1, 56.8, 57.2, 63.8, 118.2, 127.6, 128.5 (2 C), 128.8 (2 C), 134.9, 137.6, 170.3. IR (film): $v=3300,3065,2955,2847$, $1647,1471,1439,1385,1360,1253,1099,917,837,777,701 \mathrm{~cm}^{-1}$. MS (ES): $417[\mathrm{M}+1]^{+}(100 \%)$. Anal. calcd for $\mathrm{C}_{24} \mathrm{H}_{40} \mathrm{~N}_{2} \mathrm{O}_{2} \mathrm{Si}(416.3)$ : C, 69.18; H, 9.68; N, 6.72; S, 6.74; found: C, 68.97; H, 9.98; N, 6.49; S, 6.63 .

(-)-(3S,5R,6S)-3-Allyl-1-benzyl-6-[(t-butyldimethylsilyloxy)methyl]-5-(i-propyl)piperazin-2-one,

13d. Data for 13d: $\boldsymbol{R}_{\mathbf{f}}=0.32(20 \%$ EtOAc-hexane $) . \quad[\alpha]^{\mathbf{2 0}}{ }_{\mathbf{D}}=-11.8(c=0.11) .{ }^{1} \mathbf{H}$ NMR $(400 \mathrm{MHz})$ $\delta$ 0.00-0.05 (m, $6 \mathrm{H}), 0.74(\mathrm{~d}, 6 \mathrm{H}, J=6.6 \mathrm{~Hz}), 0.87(\mathrm{~s}, 9 \mathrm{H}), 1.55(\mathrm{br} \mathrm{s}, 1 \mathrm{H}), 1.60(\mathrm{~m}, 1 \mathrm{H}), 2.43(\mathrm{~m}, 1$ H), $2.78(\mathrm{~m}, 1 \mathrm{H}), 2.81(\mathrm{t}, 1 \mathrm{H}, J=5.2 \mathrm{~Hz}), 3.16(\mathrm{dt}, 1 \mathrm{H}, J=6.0,3.8 \mathrm{~Hz}), 3.31(\mathrm{dd}, 1 \mathrm{H}, J=8.3,3.9$ Hz), $3.45(\mathrm{dd}, 1 \mathrm{H}, J=10.7,3.6 \mathrm{~Hz}), 3.67(\mathrm{dd}, 1 \mathrm{H}, J=10.6,4.0 \mathrm{~Hz}), 4.00(\mathrm{~d}, 1 \mathrm{H}, J=14.8 \mathrm{~Hz}), 5.10$ (ap d, $1 \mathrm{H}, J=10.2 \mathrm{~Hz}$ ), 5.16 (ap d, $1 \mathrm{H}, J=17.0 \mathrm{~Hz}$ ), $5.33(\mathrm{~d}, 1 \mathrm{H}, J=15.0 \mathrm{~Hz}$ ), 5.87 (ddt, $1 \mathrm{H}, J=$ 17.0, 10.2, $6.9 \mathrm{~Hz}), 7.21-7.31$ (m, $5 \mathrm{H})$. DNOE between H-3 / $1 \mathrm{H} \mathrm{CH}_{2}$ allyl: 4.8\%; H-3 / $1 \mathrm{H} \mathrm{CH}_{2}$ allyl: 1.8\%; H-6 / CH iPr: 1.5\%; H-6 / 1 H CH $2 \mathrm{O}: 1.9 \%$; H-6 / $\mathrm{CH}_{3}: 3.9 \%$; H-6 / $1 \mathrm{H} \mathrm{CH}_{2} \mathrm{Ph}: 0.5 \%$; $\mathrm{CH}$ $i \operatorname{Pr} / \mathrm{H}-6: 0.7 \%$; CH $i \operatorname{Pr} / \mathrm{H}-5: 0.8 \%$; CH $i \operatorname{Pr} / \mathrm{CH}_{3}: 4.0 \% .{ }^{13} \mathrm{C}$ NMR (100 MHz) $\delta-5.6,-5.5,17.4$, 18.2, 19.3, 25.8 (3 C), 31.2, 36.0, 47.4, 56.5, 58.4, 59.0, 63.3, 117.7, 127.3, 128.2 (2 C), 128.5 (2 C), 135.6, 137.5, 173.2. IR (film): $v=3375,2925,2855,1736,1658,1462,1374,1258,1107,836,778$, $700 \mathrm{~cm}^{-1}$. MS (ES): $417[\mathrm{M}+1]^{+}(100 \%)$. Anal. calcd for $\mathrm{C}_{24} \mathrm{H}_{40} \mathrm{~N}_{2} \mathrm{O}_{2} \mathrm{Si}(416.3)$ : C, 69.18; H, 9.68; N, 6.72; S, 6.74; found: C, 69.09; H, 9.79; N, 6.82; S, 6.54 .

\section{(+)-(3R,5S,6R)-1-Benzyl-6-[(t-butyldimethylsilyloxy)methyl]-3-(1,1-dimethylallyl)-5-(i-propyl)} piperazin-2-one, 14e and (3R,5S,6R)-1-benzyl-6-[(t-butyldimethylsilyloxy)methyl]-3-(3-methylbut2-enyl)-5-( $i$-propyl)piperazin-2-one, 14f. From 9c (24 mg, $0.064 \mathrm{mmol})$ and 1-bromo-3-methyl but-2ene (14 mg, $11 \mathrm{~mL}, 0.096 \mathrm{mmol})$ was obtained after work-up a mixture (65:35) of 14e and 14f. After chromatography (5-10\% Et $2 \mathrm{O}$-hexane) $\mathbf{1 4 e}(13 \mathrm{mg}, 0.029 \mathrm{mmol}, 46 \%)$ and $\mathbf{1 4 f}(7 \mathrm{mg}, 0.016 \mathrm{mmol}$, 
$25 \%)$ were isolated as colorless oils. Data for 14e: $\boldsymbol{R}_{\mathbf{f}}=0.27\left(20 \% \mathrm{Et}_{2} \mathrm{O}-\right.$ hexane $) .[\alpha]^{\mathbf{2 0}}{ }_{\mathbf{D}}=+90.4(c=$ 0.23). ${ }^{1}$ H NMR (300 MHz) $\delta 0.05(\mathrm{~s}, 6 \mathrm{H}), 0.26(\mathrm{~d}, 3 \mathrm{H}, J=6.3 \mathrm{~Hz}), 0.80(\mathrm{~d}, 3 \mathrm{H}, J=6.6 \mathrm{~Hz}), 0.88(\mathrm{~s}$, $9 \mathrm{H}), 1.15(\mathrm{~s}, 3 \mathrm{H}), 1.25(\mathrm{~s}, 3 \mathrm{H}), 1.51(\mathrm{~m}, 1 \mathrm{H}), 1.64($ br s, $1 \mathrm{H}), 2.33($ ap d, $1 \mathrm{H}, J=10.5 \mathrm{~Hz}), 3.20$ (ddd, $1 \mathrm{H}, J=7.8,5.0,1.1 \mathrm{~Hz}), 3.30(\mathrm{~s}, 1 \mathrm{H}), 3.78(\mathrm{dd}, 1 \mathrm{H}, J=9.4,5.2 \mathrm{~Hz}), 3.79(\mathrm{~d}, 1 \mathrm{H}, J=14.4$ $\mathrm{Hz}), 3.90(\mathrm{dd}, 1 \mathrm{H}, J=9.8,8.1 \mathrm{~Hz}), 5.02(\mathrm{dd}, 1 \mathrm{H}, J=18.1,1.5 \mathrm{~Hz}), 5.03(\mathrm{~d}, 1 \mathrm{H}, J=10.3,1.5 \mathrm{~Hz})$, $5.42(\mathrm{~d}, 1 \mathrm{H}, J=14.2 \mathrm{~Hz}), 5.90(\mathrm{dd}, 1 \mathrm{H}, J=17.8,10.3 \mathrm{~Hz}), 7.22-7.33(\mathrm{~m}, 5 \mathrm{H}) .2 \mathrm{D}-\mathrm{NOESY}$ showed cross points between: $\mathrm{H}-3 / 2 \mathrm{CH}_{3}(i \mathrm{Pr}, 0.26$ and $0.88 \mathrm{ppm}), \mathrm{H}-3 / \mathrm{CH}(i \mathrm{Pr}) .{ }^{13} \mathbf{C}$ NMR $(75 \mathrm{MHz}) \delta-5.3$ (2 C), 18.2 (1 C), 19.3 (2 C), 22.6, 25.2, 25.4, 25.9 (3 C), 41.5, 48.8, 56.8, 57.4, 61.4, 63.8, 112.1, 127.5, 128.5 (2 C), 129.1 (2 C), 137.9, 146.4, 169.9. IR (film): $v=3391,2956,2920,2862,1646$, 1443, 1256, 1230, 1089, 840, $771 \mathrm{~cm}^{-1}$. MS (ES): $911[2 \mathrm{M}+\mathrm{Na}]^{+}, 445[\mathrm{M}+1]^{+}(100 \%)$. Anal. calcd for $\mathrm{C}_{26} \mathrm{H}_{44} \mathrm{~N}_{2} \mathrm{O}_{2} \mathrm{Si}$ (444.7): C, 70.22; H, 9.97; N, 6.30; O, 7.20; Si, 6.32; found: C, 69.99; H, 9.84; N, 6.29. Partial data of 14f: $R_{\mathrm{f}}=0.10\left(20 \% \mathrm{Et}_{2} \mathrm{O}\right.$-hexane) ${ }^{1} \mathbf{H}$ NMR $(200 \mathrm{MHz}) \delta 0.05(\mathrm{~m}, 6 \mathrm{H}), 0.36(\mathrm{~d}$, $6 \mathrm{H}, J=6.6 \mathrm{~Hz}), 0.86(\mathrm{~m}, 12 \mathrm{H}), 1.60(\mathrm{~m}, 1 \mathrm{H}), 1.66(\mathrm{~s}, 3 \mathrm{H}), 1.72(\mathrm{~s}, 3 \mathrm{H}), 2.43(\mathrm{~m}, 2 \mathrm{H}), 2.57(\mathrm{~m}, 1$ H), $3.25(\mathrm{~m}, 1 \mathrm{H}), 3.47(\mathrm{~m}, 1 \mathrm{H}), 3.73(\mathrm{dd}, 1 \mathrm{H}, J=10.0,4.5 \mathrm{~Hz}), 3.79(\mathrm{dd}, 1 \mathrm{H}, J=10.6,4.0 \mathrm{~Hz}), 3.85$ $(\mathrm{d}, 1 \mathrm{H}, J=14.4 \mathrm{~Hz}), 5.12(\mathrm{~m}, 1 \mathrm{H}), 5.42(\mathrm{~d}, 1 \mathrm{H}, J=14.4 \mathrm{~Hz}), 7.28(\mathrm{~m}, 5 \mathrm{H}) . \mathbf{I R}($ film): $v=3405$, 2956, 2920, 2848, 1657, 1465, 1451, 1162, 1060, $956 \mathrm{~cm}^{-1}$. MS (ES): $445[\mathrm{M}+1]^{+}, 331[\mathrm{M}-$ TBDMS +2$]^{+}(100 \%)$.

\section{References}

(1) Viso, A.; Fernández de la Pradilla, R.; García, A.; Guerrero-Strachan, C.; Alonso, M.; Flores, A.; Martínez-Ripoll, M.; Fonseca, I.; André, I.; Rodríguez, A. Chem. Eur. J. 2003, 9, 2867-2876.

(2) Viso, A.; Fernández de la Pradilla, R.; López-Rodríguez, M. L.; García, A.; Flores, A.; Alonso, M.; J. Org. Chem. 2004, 69, 1542.

(3) Martínez, E. J.; Corey, E. J. Org. Lett. 2000, 2, 993-996.

(4) Hou, X. L.; Zheng, X. L.; Dai, L. X. Tetrahedron Lett. 1998, 39, 6949-6952. 
(5) Laschat, S.; Kunz, H. J. Org. Chem. 1991, 56, 5883-5889.

(6) Basile, T.; Bocoum, A.; Savoia, D.; Umani-Ronchi, A. J. Org. Chem. 1994, 59, 7766-7773. 